\title{
Cyanobacteria of Greece: an annotated checklist
}

\author{
Spyros Gkelis ${ }^{\ddagger}$, Iordanis Ourailidis ${ }^{\ddagger}$, Manthos Panou $\ddagger$, Nikos Pappas ${ }^{\ddagger}$ \\ ‡ Department of Botany, School of Biology, Aristotle University of Thessaloniki, Thessaloniki, Greece
}

Corresponding author: Spyros Gkelis (sgkelis@bio.auth.gr)

Academic editor: Vasilis Gerovasileiou

Received: 04 Aug 2016 | Accepted: 24 Aug 2016 | Published: 01 Nov 2016

Citation: Gkelis S, Ourailidis I, Panou M, Pappas N (2016) Cyanobacteria of Greece: an annotated checklist.

Biodiversity Data Journal 4: e10084. https://doi.org/10.3897/BDJ.4.e10084

\section{Abstract}

\section{Background}

The checklist of Greek Cyanobacteria was created in the framework of the Greek Taxon Information System (GTIS), an initiative of the LifeWatchGreece Research Infrastructure (ESFRI) that has resumed efforts to compile a complete checklist of species reported from Greece. This list was created from exhaustive search of the scientific literature of the last 60 years. All records of taxa known to occur in Greece were taxonomically updated.

\section{New information}

The checklist of Greek Cyanobacteria comprises 543 species, classified in 130 genera, 41 families, and 8 orders. The orders Synechococcales and Oscillatoriales have the highest number of species (158 and 153 species, respectively), whereas these two orders along with Nostocales and Chroococcales cover $93 \%$ of the known Greek cyanobacteria species. It is worth mentioning that 18 species have been initially described from Greek habitats. The marine epilithic Ammatoidea aegea described from Saronikos Gulf is considered endemic to this area. Our bibliographic review shows that Greece hosts a high diversity of cyanobacteria, suggesting that the Mediterranean area is also a hot spot for microbes. 


\section{Keywords}

blue-green algae, Cyanophyceae, bacteria, microorganisms, Aegean Sea, freshwater, marine, checklist, biodiversity, Mediterranean

\section{Introduction}

The history of natural science is directly linked to Greek civilization, since the older written references to plants, animals, and algae are found in the Homeric Epics dating back to the $8^{\text {th }}$ century BC; the first record of algae in the Western literature appears in Homer's Iliad: "the dark waves uprear their heads and scatter their sea-wrack (phycos) in all directions" [lliad IX.1 translated by Batler (1998)]. The Greek philosopher, and first marine biologist, Aristotle, who has established scientific knowledge for many animal and plant taxa (see Voultsiadou 2007) reported "red snow" in $4^{\text {th }}$ century BC: "after lying for some time, snow turns slightly red" (Historia Animalium 552b8 translated by Peck 1970); this is considered as one of the oldest records of snow algae [unicellular Chlorophyta, like Chlamydomonas or Chloromonas according to Duval et al. (1999)].

In modern times, research on Greek microflora, Cyanobacteria included, started in the $20^{\text {th }}$ century with the studies of freshwater algae by Stanković (1931) and Skuja (1937). In the subsequent two decades, no published work in microflora can be traced until the limnological survey by Ananiadis (1956). During the 1960s Konstantinos Anagnostidis performs two extensive studies of Cyanobacteria from thermal springs (Anagnostidis 1961) and sulphur-communities (Sulphuretum) in marine and freshwater habitats of Greece (Anagnostidis 1968). These taxonomic studies revealed the occurrence of a great number of cyanobacteria taxa, led to the description of several new species (e.g. Geitlerinema apolloniae Anagnostidis, 2001; Leptolyngbya phormidioides (Anagnostidis) Anagnostidis \& Komárek, 1988; Pseudanabaena lonchoides Anagnostidis, 1961; Pseudophormidium battersii (Gomont) Anagnostidis, 2001; Borzia periklei Anagnostidis in (Anagnostidis and Komárek 1988)), and were later widely used to establish the modern classification system of Cyanobacteria (Anagnostidis and Komárek 1985, Anagnostidis and Komárek 1988, Anagnostidis and Komárek 1990, Komárek and Anagnostidis 1986, Komárek and Anagnostidis 1989). Although a lot of the valuable taxonomic, ecological, and biogeographical information recorded in Anagnostidis' monographs is now contained in the "Süsswasserflora von Mitteleuropa" series (Komárek 2013, Komárek and Anagnostidis 1999, Komárek and Anagnostidis 2005, Komárek 2013), many details of his work remain unavailable to the broader scientific community since they were published in Greek.

The systematic research on Greek Cyanobacteria started in the 1980s with a series of publications on cyanobacterial taxonomy and species composition (Anagnostidis and Economou-Amilli 1978, Anagnostidis and Economou-Amilli 1980, Anagnostidis et al. 1981, Anagnostidis et al. 1982, Overbeck et al. 1982, Anagnostidis et al. 1983, Anagnostidis and Pantazidou 1985, Anagnostidis and Pantazidou 1988a, Anagnostidis and Pantazidou 1988b, Anagnostidis and Pantazidou 1988c, Hindak and Moustaka 1988, Anagnostidis et 
al. 1988a, Anagnostidis and Pantazidou 1991a, Anagnostidis and Pantazidou 1991b, Anagnostidis and Roussomoustakaki 1991, Tryfon 1996), as well as on population dynamics (Moustaka-Gouni 1988, Moustaka-Gouni and Nikolaidis 1990, Moustaka-Gouni and Nikolaidis 1992, Tafas and Economou-Amilli 1997, Tryfon et al. 1997, Montesanto et al. 1999, Moustaka-Gouni et al. 2000, Temponeras et al. 2000, Vardaka et al. 2000) regarding both the freshwater and marine waterbodies. The recent research on cyanobacterial diversity in Greece comprises polyphasic or multidisciplinary studies of cyanobacteria strains (Gkelis et al. 2005b, Lamprinou et al. 2011, Lamprinou et al. 2013a, Lamprinou et al. 2013b, Gkelis et al. 2015b, Gkelis and Panou 2016, Bravakos et al. 2016), natural populations (Moustaka-Gouni et al. 2009, Moustaka-Gouni et al. 2010, Papadimitriou et al. 2013, Gkelis and Zaoutsos 2014, Gkelis et al. 2014, Gkelis et al. 2015a), as well as ecological works (e.g. Lamprinou et al. 2009, Katsiapi et al. 2011, Katsiapi et al. 2012).

Despite the increased research efforts, knowledge of diversity and distribution of Cyanobacteria in Greece is still inconsistent since no list on local cyanobacterial flora has ever been published. Modern biodiversity research faces an ever increasing rate of data generation and efforts are made to structure, aggregate, link and process these data in a meaningful way (Koureas et al. 2016). Currently, the Greek Taxon Information System (GTIS), an initiative launched by the LifeWatchGreece Research Infrastructure (ESFRI), is resuming efforts to compile a database of all species reported from Greece; a prerequisite for GTIS is to have checklists for each taxonomic group (Bailly et al. 2016). The aim of this study is to create a checklist of cyanobacterial taxa inhabiting the area of Greece on the basis of the recent taxonomic revisions.

\section{Materials and methods}

The Checklist of Greek Cyanobacteria was created in the framework of the Greek Taxon Information System (GTIS), an initiative of the LifeWatchGreece Research Infrastructure (ESFRI) that has resumed efforts to compile a complete checklist of all species reported from Greece (Bailly et al. 2016). In that publication, a methodology is described to produce Preliminary Checklists only. However, in the present case of Cyanobacteria, the status of the list for Greece was quite advanced, and the recent primary literature was exhaustively searched for this work; the present list is thus considered as an updated, annotated, and archived checklist.

The data for this study were collected through extensive search in the scientific literature of the last 60 years. The search was performed in Scopus and Web of Science using the keywords: Greece AND cyanobacteria, Greece AND phytoplankton, Greece AND cyanoprokaryota, Greece AND blue-green algae. Furthermore, the two exhaustive monographs by Anagnostidis (Anagnostidis 1961, Anagnostidis 1968) were studied and the cyanobacterial taxa were recorded. All geographical areas of Greece and all biomes (freshwater, marine, terrestrial, caves etc.) and biotic forms (planktic, benthic, periphytic, endolithic, epizoic etc.) were considered. 
The taxonomic status of the recorded cyanobacterial taxa was checked and updated, where necessary, to the currently accepted taxonomically species using Komárek and Anagnostidis (1999), Komárek and Anagnostidis (2005), Komárek (2013), and the AlgaeBase (Guiry and Guiry 2016). The species were classified to suprageneric taxa according to the latest taxonomic revisions on the basis of polyphasic methods (Komárek et al. 2014, Komárek 2016). Not valid species, species of unclear taxonomic status, and species transferred to other phyla were excluded from the list. Also, infraspecific taxa that are not valid or need revision were not included in the list. The first publication mentioning a taxon's occurrence in Greece and the original name used in that record is given under Notes and Nomeclature, respectively.

\section{Data resources}

The data collected were published in Global Biodiversity Information Facility (GBIF) through the Integrated Publishers Toolkit (IPT) according to the Darwin Core Archive biodiversity informatics data standard and are made publicly available through GBIF (UUID: $655027 \mathrm{fc}-$ 76ea-447a-a443-f36dd2e853d7, DOI: 10.15468/lkj0mr).

\section{List of Cyanobacteria known to occur in Greece}

\section{Ammatoidea aegaea Anagnostidis \& Pantazidou, 1991}

Nomenclature:

Ammatoidea aegea

Notes: Anagnostidis and Pantazidou 1991b

Anabaena cf. cylindrica Lemmermann, 1896

Nomenclature:

Anabaena cf. cylindrica

Notes: Gkelis et al. 2015b

Anabaena inaequalis Bornet \& Flahault, 1888

Nomenclature:

Anabaena inaequalis

Notes: Tafas and Economou-Amilli 1997 
Anabaena laxa A. Braun in Bornet \& Falhault, 1888

Nomenclature:

Anabaena laxa

Notes: Anagnostidis 1961

Anabaena oscillarioides Bory ex Bornet \& Flahault, 1888

Nomenclature:

Anabaena oscillarioides

Notes: Anagnostidis 1961

Anabaena perturbata H. Hill, 1976

Nomenclature:

Anabaena pertubata

Notes: Moustaka 1988

Anabaena planctonica Brunnthaler, 1903

Nomenclature:

Anabaena solitaria f. planctonica

Notes: Moustaka 1988

Anabaena sphaerica Bornet \& Flahault, 1888

Nomenclature:

Anabaena sphaerica

Notes: Tafas and Economou-Amilli 1997

Anabaena torulosa Lagerheim ex Bornet \& Flahault, 1888

Nomenclature:

Anabaena torulosa

Notes: Anagnostidis 1961

Anabaenopsis arnoldii Aptekar, 1926

Nomenclature:

Anabaenopsis arnoldii 
Notes: Moustaka-Gouni et al. 2007

Anabaenopsis cf. circularis (G. S. West) Woloszynska \& V.Miller, 1923

Nomenclature:

Anabaenopsis cf. elenkinii f. circularis

Notes: Moustaka 1988

Anabaenopsis circularis (G. S. West) Woloszynska \& V.Miller, 1923

Nomenclature:

Anabaenopsis cunningtonii

Notes: Vardaka et al. 2005

Anabaenopsis elenkinii V. V. Miller, 1923

Nomenclature:

Anabaenopsis elenkinii

Notes: Vardaka et al. 2005

Anabaenopsis milleri Woronichin, 1929

Nomenclature:

Anabaenopsis milleri

Notes: Lanaras et al. 1989

Anabaenopsis tanganyikae (G. S. West) Woloszynska \& V.Miller, 1923

Nomenclature:

Anabaenopsis tanganyikae

Notes: Hindak and Moustaka 1988

Anathece clathrata (W. \& G. S. West) Komárek, Kastovsky \& Jezberová, 2011 Nomenclature:

Aphanothece clathrata

Notes: Anagnostidis 1968 
Anathece endophytica (W. \& G. S. West) Komárek, Kastovsky \& Jezberová, 2011

Nomenclature:

Aphanothece nidulans var. endophytica

Notes: Hindak and Moustaka 1988

Anathece minutissima (West) Komárek, Kastovsky \& Jezberová, 2011

Nomenclature:

Aphanothece minutissima

Notes: Tryfon et al. 1997

Aphanizomenon favaloroi S.H.Otaño, 2012

Nomenclature:

Aphanizomenon favaloroi

Notes: Moustaka-Gouni et al. 2016

Aphanizomenon flos-aquae Ralfs ex Bornet \& Flahault, 1886

Nomenclature:

Aphanizomenon flos-aquae

Notes: Ananiadis 1956

Aphanizomenon gracile Lemmermann, 1907

Nomenclature:

Aphanizomenon gracile

Notes: Anagnostidis 1968

Aphanocapsa biformis A. Braun in P. Richter, 1879

Nomenclature:

Aphanocapsa biformis

Notes: Anagnostidis 1961 
Aphanocapsa cf. holsatica (Lemmermann) G. Cronberg \& Komárek, 1994

Nomenclature:

Microcystis cf. holsatica

Notes: Hindak and Moustaka 1988

Aphanocapsa conferta (W. \& G. S. West) Komárková-Legnerová \& Cronberg, 1994

Nomenclature:

Aphanocapsa elachista var. conferta

Notes: Tryfon et al. 1997

Aphanocapsa delicatissima W. \& G. S. West, 1912

Nomenclature:

Aphanocapsa delicatissima

Notes: Moustaka-Gouni 1988

Aphanocapsa elachista W. \& G. S. West, 1912

Nomenclature:

Aphanocapsa elachista

Notes: Anagnostidis 1968

Aphanocapsa fusco-lutea Hansgirg, 1892

Nomenclature:

Aphanocapsa fusco-lutea

Notes: Lamprinou et al. 2012

Aphanocapsa grevillei (Berkeley) Rabenhorst, 1865

Nomenclature:

Aphanocapsa grevillei

Notes: Anagnostidis 1961 
Aphanocapsa incerta (Lemmermann) G. Cronberg \& Komárek, 1994 Nomenclature:

Aphanocapsa incerta

Notes: Tryfon et al. 1996

Aphanocapsa marina Hansgirg, 1892

Nomenclature:

Aphanocapsa marina

Notes: Anagnostidis 1968

Aphanocapsa muscicola (Meneghini) Wille, 1919

Nomenclature:

Aphanocapsa muscicola

Notes: Anagnostidis 1961

Aphanocapsa parietina (Nägeli ex Kützing) Nägeli, 1849

Nomenclature:

Aphanocapsa parientina

Notes: Lamprinou et al. 2012

Aphanocapsa raspaigellae (Hauck) Frémy in Feldmann, 1933

Nomenclature:

Aphanocapsa raspaigellae

Notes: Anagnostidis 1968

Aphanocapsa rivularis (Carmichael) Rabenhorst, 1865

Nomenclature:

Aphanocapsa anodontae

Notes: Anagnostidis 1968

Aphanocapsa salina Woronichin (Voronichin), 1929

Nomenclature:

Aphanocapsa salina 
Notes: Anagnostidis and Golubic 1966

Aphanocapsa sesciacensis Frémy, 1928

Nomenclature:

Aphanocapsa sescianensis

Notes: Anagnostidis and Golubic 1966

Aphanocapsa thermalis Brügger, 1863

Nomenclature:

Aphanocapsa thermalis

Notes: Anagnostidis 1961

Aphanothece castagnei (Kützing) Rabenhorst, 1865

Nomenclature:

Aphanothece castagnei

Notes: Anagnostidis 1961

Aphanothece cf. rubra Liebetanz, 1926

Nomenclature:

Aphanothece cf. rubra

Notes: Lamprinou et al. 2012

Aphanothece cf. rufescens Hansgrig, 1892

Nomenclature:

Aphanothece cf. rufescens

Notes: Lamprinou et al. 2012

Aphanothece cf. salina Elenkin \& Danilov, 1915

Nomenclature:

Aphanothece cf. salina

Notes: Anagnostidis and Roussomoustakaki 1991 
Aphanothece marina (Ercegovic) Komárek \& Anagnostidis, 1995

Nomenclature:

Synechococcus marinus

Notes: Anagnostidis 1968

Aphanothece microscopica Nägeli, 1849

Nomenclature:

Aphanothece microscopica

Notes: Anagnostidis 1968

Aphanothece microspora (Meneghini) Rabenhorst, 1863

Nomenclature:

Aphanothece microspora

Notes: Anagnostidis 1968

Aphanothece nidulans Richter in Wittrock \& Nordstedt, 1884

Nomenclature:

Aphanothece nidulans

Notes: Anagnostidis 1961

Aphanothece pallida (Kützing) Rabenhorst, 1863

Nomenclature:

Aphanothece pallida

Notes: Lamprinou et al. 2012

Aphanothece saxicola Nägeli, 1849

Nomenclature:

Aphanothece saxicola

Notes: Anagnostidis 1968

Aphanothece stagnina (Sprengel) A. Braun in Rabenhorst, 1863

Nomenclature:

Aphanothece stagnina 
Notes: Anagnostidis 1968

Arthrospira fusiformis (Voronikhin) Komárek \& J. W. G. Lund, 1990

Nomenclature:

Arthrospira fusiformis

Notes: Moustaka-Gouni et al. 2007

Arthrospira platensis Gomont, 1892

Nomenclature:

Arthrospira platensis

Notes: Vardaka et al. 2005

Asterocapsa aerophytica F. Lederer, 2000

Nomenclature:

Asterocapsa aerophytica

Notes: Lamprinou et al. 2012

Asterocapsa divina Komárek, 1993

Nomenclature:

Asterocapsa divina

Notes: Lamprinou et al. 2012

Asterocapsa jilinica H. X. Xiao, 2000

Nomenclature:

Asterocapsa jilinica

Notes: Lamprinou et al. 2012

Asterocapsa sinica Liang \& Chen, 1985

Nomenclature:

Asterocapsa sinica

Notes: Lamprinou et al. 2012 
Blennothrix brebissonii (Kützing ex Gomont) Anagnostidis \& Komárek, 1988 Nomenclature:

Hydrocoleum brebissonii

Notes: Anagnostidis 1968

Blennothrix glutinosa (Gomont ex Gomont) Anagnostidis \& Komárek, 2001 Nomenclature:

Hydrocoleum glutinosum

Notes: Anagnostidis 1968

Blennothrix heterotricha (Gomont ex Gomont) Anagnostidis \& Komárek, 1988 Nomenclature:

Hydrocoleum heterotrichum

Notes: Anagnostidis 1968

Blennothrix lyngbyacea (Kützing ex Gomont) Anagnostidis \& Komárek, 1988 Nomenclature:

Hydrocoleum lyngbyaceum

Notes: Anagnostidis and Golubic 1966

Borzia periklei Anagnostidis in Anagnostidis \& Komárek, 1988

Nomenclature:

Borzia trilocularis

Notes: Anagnostidis 1977

Borzia starkii Schiller, 1954

Nomenclature:

Borzia starkii

Notes: Anagnostidis 1977

Borzia susedana Ercegovic, 1925

Nomenclature:

Borzia susedana 
Notes: Anagnostidis 1977

Borzia trilocularis Cohn ex Gomont, 1892

Nomenclature:

Borzia trilocularis

Notes: Anagnostidis 1968

Brachytrichia quoyi Bornet \& Flahault, 1886

Nomenclature:

Brachytrichia quiyi

Notes: Anagnostidis 1968

Calothrix aeruginea Thuret ex Bornet \& Flahault, 1886

Nomenclature:

Calothrix aeruginea

Notes: Anagnostidis 1968

Calothrix braunii Bornet \& Flahault, 1886

Nomenclature:

Calothrix braunii

Notes: Anagnostidis 1968

Calothrix cf. fusca Bornet \& Flahault, 1886

Nomenclature:

Calothrix cf. fusca

Notes: Lamprinou et al. 2012

Calothrix confervicola C. Agardh ex Bornet \& Flahault, 1886

Nomenclature:

Calothrix confervicola

Notes: Anagnostidis 1968 
Calothrix contarenii Bornet \& Flahault, 1886

Nomenclature:

Calothrix contarenii

Notes: Anagnostidis 1968

Calothrix javanica De Wildeman, 1897

Nomenclature:

Calothrix javanica

Notes: Anagnostidis et al. 1981

Calothrix marchica Lemmermann, 1914

Nomenclature:

Calothrix marchica

Notes: Anagnostidis et al. 1981

Calothrix parietina Thuret ex Bornet \& Flahault, 1886

Nomenclature:

Calothrix parietina

Notes: Anagnostidis 1968

Calothrix pulvinata C. Agardh ex Bornet \& Flahault, 1886

Nomenclature:

Calothrix pulvinata

Notes: Anagnostidis and Golubic 1966

Calothrix scopulorum C. Agardh ex Bornet \& Flahault, 1886 Nomenclature:

Calothrix scopulorum

Notes: Anagnostidis and Golubic 1966

Calothrix stagnalis Gomont, 1895

Nomenclature:

Calothrix stagnalis 
Notes: Anagnostidis et al. 1981

Calothrix thermalis Hasngirg ex Bornet \& Flahault, 1886

Nomenclature:

Calothrix thermalis

Notes: Anagnostidis 1968

Chamaesiphon confervicola A. Braun in Rabenhorst, 1865

Nomenclature:

Chamaesiphon curvatus

Notes: Anagnostidis 1968

Chamaesiphon geitleri A. Braun in Rabenhorst, 1865

Nomenclature:

Chamaesiphon geitleri

Notes: Anagnostidis 1968

Chamaesiphon incrustans Grunow in Rabenhorst, 1865

Nomenclature:

Chamaesiphon incrustans

Notes: Anagnostidis 1968

Chamaesiphon polonicus (Rostafinski) Hansgirg, 1893

Nomenclature:

Chamaesiphon polonicus

Notes: Anagnostidis 1968

Chamaesiphon polymorphus Geitler, 1925

Nomenclature:

Chamaesiphon polymorphus

Notes: Anagnostidis 1968 
Chlorogloea microcystoides Geitler, 1926

Nomenclature:

Chlorogloea microcystoides

Notes: Anagnostidis 1961

Chlorogloea novacekii Komárek \& Montejano, 1994

Nomenclature:

Chlorogloea novacekii

Notes: Lamprinou et al. 2012

Chlorogloea rivularis (Hansgirg) Komárek \& Anagnostidis, 1995

Nomenclature:

Xenococcus rivularis

Notes: Anagnostidis 1968

Chroococcidiopsis doonensis R. B. Singh, 1968

Nomenclature:

Chroococcidiopsis doonensis

Notes: Lamprinou et al. 2009

Chroococcidiopsis kashayi Friedmann, 1961

Nomenclature:

Chroococcidiopsis kashaii

Notes: Lamprinou et al. 2012

Chroococcidiopsis thermalis Geitler, 1933

Nomenclature:

Chroococcidiopsis thermalis

Notes: Anagnostidis and Pantazidou 1988a

Chroococcidium gelatinosum Geitler, 1933

Nomenclature:

Chroococcidium gelatinosum 
Notes: Metaxatos et al. 2003

Chroococcus cf. aphanocapsoides Skuja ex Joosten, 2006

Nomenclature:

Chroococcus cf. aphanocapsoides

Notes: Lamprinou et al. 2012

Chroococcus cf. subsphaericus N. L. Gardner, 1927

Nomenclature:

Chroococcus cf. subsphaericus

Notes: Lamprinou et al. 2012

Chroococcus cohaerens (Brébisson) Nägeli, 1849

Nomenclature:

Chroococcus cohaerens

Notes: Lamprinou et al. 2009

Chroococcus dispersus (Keissler) Lemmermann, 1904

Nomenclature:

Chroococcus dispersus

Notes: Anagnostidis 1968

Chroococcus helveticus Nägeli, 1849

Nomenclature:

Chroococcus helveticus

Notes: Anagnostidis et al. 1981

Chroococcus lithophilus Ercegovic, 1925

Nomenclature:

Chroococcus lithophilus

Notes: Lamprinou et al. 2012 
Chroococcus microscopicus Komárková-Legnerová \& G. Cronberg, 1994

Nomenclature:

Chroococcus microscopicus

Notes: Tryfon et al. 1997

Chroococcus minor (Kützing) Nägeli, 1849

Nomenclature:

Chroococcus minor

Notes: Anagnostidis 1961

Chroococcus minutus (Kützing) Nägeli, 1849

Nomenclature:

Chroococcus minutus

Notes: Anagnostidis 1961

Chroococcus spelaeus Ercegovic, 1925

Nomenclature:

Chroococcus spelaeus

Notes: Lamprinou et al. 2009

Chroococcus subnudus (Hansgirg) G. Cronberg \& J. Komárek, 1994

Nomenclature:

Chroococcus subnudus

Notes: Lamprinou et al. 2012

Chroococcus tenax (Kirchner) Hieronymus, 1892

Nomenclature:

Chroococcus tenax

Notes: Lamprinou et al. 2009

Chroococcus thermalis (Meneghini) Nägeli, 1849

Nomenclature:

Chroococcus thermalis 
Notes: Radea et al. 2010

Chroococcus turgidus (Kützing) Nägeli, 1849

Nomenclature:

Chroococcus turgidus

Notes: Anagnostidis 1961

Chroococcus turicensis (Nägeli) Hansgirg, 1887

Nomenclature:

Chroococcus turicensis

Notes: Lamprinou et al. 2012

Chroococcus varius A. Braun in Rabenhorst, 1876

Nomenclature:

Chroococcus varius

Notes: Anagnostidis 1968

Chroococcus westii Boye-Petersen, 1923

Nomenclature:

Chroococcus westii

Notes: Lamprinou et al. 2012

Chrysosporum bergii (Ostenfeld) E. Zapomelová, O. Skácelová, P. Pumann, R. Kopp \& E. Janecek, 2012

Nomenclature:

Anabaena bergii

Notes: Katsiapi et al. 2011

Chrysosporum ovalisporum (Forti) E. Zapomelová, O. Skácelová, P. Pumann, R. Kopp \& E. Janecek, 2012

Nomenclature:

Aphanizomenon ovalisporum

Notes: Gkelis et al. 2005a 
Clastidium rivulare (Hansgirg) Hansgirg, 1892

Nomenclature:

Clastidium rivulare

Notes: Anagnostidis 1968

Clastidium setigerum 0. Kirchner, 1880

Nomenclature:

Clastidium setigerum

Notes: Anagnostidis 1968

Coelosphaerium kützingianum Nägeli, 1849

Nomenclature:

Coelosphaerium minutissimum

Notes: Anagnostidis 1968

Coleofasciculus chthonoplastes (Thuret ex Gomont) M. Siegesmund, J. R. Johansen \& T. Friedl in Siegesmund et al. 2008

Nomenclature:

Microcoleus chtonoplastes

Notes: Anagnostidis 1968

Cuspidothrix issatschenkoi (Usachev) P. Rajaniemi, Komárek, R. Willame, P. Hrouzek, K. Kastovská, L. Hoffmann \& K. Sivonen, 2005

Nomenclature:

Aphanizomenon issatschenkoi

Notes: Moustaka-Gouni 1988

Cyanobacterium cedrorum (Sauvageau) Komárek, Kopecky \& Cepák, 1999

Nomenclature:

Synechococcus cedrorum

Notes: Anagnostidis 1968 
Cyanobacterium minervae (J. J. Copeland) Komárek, Kopecky \& Cepák, 1999

Nomenclature:

Synechococcus minervae

Notes: Anagnostidis 1961

Cyanocatena planctonica Hindák, 1975

Nomenclature:

Cyanocatena planctonica

Notes: Moustaka-Gouni 1988

Cyanodictyon imperfectum Cronberg \& Weibull, 1981

Nomenclature:

Cyanodictyon imperfectum

Notes: Moustaka-Gouni and Nikolaidis 1990

Cyanodictyon planctonicum B. A. Mayer, 1994

Nomenclature:

Cyanodictyon planctonicum

Notes: Tryfon et al. 1996

Cyanodictyon reticulatum (Lemmermann) Geitler, 1925

Nomenclature:

Cyanodictyon reticulatum

Notes: Danielidis et al. 1996

Cyanogranis ferruginea (F. Wawrik) Hindák ex Hindák in Joosen, 2006

Nomenclature:

Cyanogranis ferruginea

Notes: Moustaka-Gouni 1988

Cyanosaccus aegaeus Anagnostidis \& Pantazidou, 1985

Nomenclature:

Cyanosaccus aegaeus 
Notes: Anagnostidis and Pantazidou 1985

Cyanosaccus atticus Anagnostidis \& Pantazidou, 1988

Nomenclature:

Cyanosaccus atticus

Notes: Anagnostidis and Pantazidou 1988b

Cyanosarcina cf. burmensis (Skuja) Kovácik, 1988

Nomenclature:

Cyanosarcina cf. burmensis

Notes: Lamprinou et al. 2012

Cyanosarcina cf. spectabilis (Geitler) Kovácik, 1988

Nomenclature:

Cyanosarcina cf. spectabilis

Notes: Lamprinou et al. 2012

Cyanosarcina parthenonensis Anagnostidis in Anagnostidis \& Pantazidou, 1991

Nomenclature:

Cyanosarcina parthenonensis

Notes: Lamprinou et al. 2012

Cyanosarcina thalassia Anagnostidis \& Pantazidou, 1991

Nomenclature:

Cyanosarcina thalassia

Notes: Anagnostidis and Pantazidou 1991a

Cyanostylon microcystoides Geitler, 1928

Nomenclature:

Cyanostylon microcystoides

Notes: Anagnostidis 1968 
Cyanostylon plancticum Hindák, 1988

Nomenclature:

Cyanostylon plancticum

Notes: Hindák 1988

Cyanothece aeruginosa (Nägeli) Komárek 1976

Nomenclature:

Synechococcus aeruginosus

Notes: Anagnostidis 1968

Cyanothece halobia Roussomoustakaki \& Anagnostidis 1991

Nomenclature:

Cyanothece halobia

Notes: Anagnostidis and Roussomoustakaki 1991

Cyanothece major (Schröter) Komárek, 1976

Nomenclature:

Synechococcus maior

Notes: Anagnostidis 1968

Cylindrospermopsis raciborskii (Woloszynska) Seenayya \& Subba Raju in Desikachary, 1972

Nomenclature:

Cylindrospermopsis raciborskii

Notes: Moustaka-Gouni 1988

Cylindrospermum licheniforme Kuitzing ex Bornet \& Flahault, 1886

Nomenclature:

Cylindrospermum licheniforme

Notes: Economou-Amilli et al. 1984 
Cylindrospermum muscicola Kützing ex Bornet \& Flahault, 1886

Nomenclature:

Cylindrospermum muscicola

Notes: Anagnostidis 1968

Dactylococcopsis echini Rosenvinge in Mortensen \& Rosenvinge, 1934

Nomenclature:

Dactylococcopsis echini

Notes: Anagnostidis 1968

Dactylococcopsis rhaphidioides Hansgirg, 1888

Nomenclature:

Dactylococcopsis rhaphidioides

Notes: Anagnostidis 1968

Dermocarpella prasina (Reinsch) Komárek \& Anagnostidis, 1995

Nomenclature:

Dermocarpa prasina

Notes: Anagnostidis 1968

Dichothrix compacta Bornet \& Flahault, 1886

Nomenclature:

Dichothrix compacta

Notes: Anagnostidis 1968

Dichothrix gypsophila Bornet \& Flahault, 1886

Nomenclature:

Dichothrix gypsophila

Notes: Anagnostidis 1968

Dolichospermum affine (Lemmermann) Wacklin, L. Hoffmann \& Komárek, 2009 Nomenclature:

Anabaena affinis 
Notes: Moustaka-Gouni and Nikolaidis 1990

Dolichospermum crassum (Lemmermann) Wacklin, L. Hoffmann \& Komárek, 2009

Nomenclature:

Anabaena crassa

Notes: Gkelis et al. 2014

Dolichospermum flos-aquae (Brébisson ex Bornet \& Flahault) P. Wacklin, L. Hoffmann \& J. Komárek, 2009

Nomenclature:

Anabaena flos-aquae

Notes: Anagnostidis 1968

Dolichospermum lemmermannii (Richter) P. Wacklin, L. Hoffmann \& J. Komárek, 2009

Nomenclature:

Anabaena lemmermannii var. minor

Notes: Tryfon et al. 1997

Dolichospermum mendotae (W. Trelease) Wacklin, L. Hoffmann \& Komárek, 2009

Nomenclature:

Anabaena mendotae

Notes: Moustaka-Gouni et al. 2000

Dolichospermum mucosum (Komárková-Legnerová \& Eloranta) Wacklin, L. Hoffmann \& Komárek, 2009

Nomenclature:

Anabaena mucosa

Notes: Tryfon et al. 1997 
Dolichospermum scheremetievii (Elenkin) Wacklin, L. Hoffmann \& Komárek, 2009

Nomenclature:

Anabaena scheremetievii

Notes: Anagnostidis 1968

Dolichospermum sigmoideum (Nygaard) Wacklin, L. Hoffmann \& Komárek, 2009

Nomenclature:

Anabaena circinalis

Notes: Moustaka-Gouni 1988

Dolichospermum solitarium (Klebahn) Wacklin, L. Hoffmann \& Komárek, 2009 Nomenclature:

Anabaena solitaria

Notes: Hindák 1993

Dolichospermum spiroides (Klebhan) Wacklin, L. Hoffmann \& Komárek, 2009 Nomenclature:

Anabaena spiroides

Notes: Anagnostidis 1968

Dolichospermum viguieri (Denis \& Frémy) Wacklin, L. Hoffmann \& Komárek, 2009

Nomenclature:

Anabaena viguieri

Notes: Lanaras et al. 1989

Entophysalis deusta (Meneghini) F. E. Drouet \& W. A. Daily 1948:

Nomenclature:

Entophysalis deusta

Notes: Anagnostidis 1968 


\section{Entophysalis granulosa Kützing 1843}

Nomenclature:

Entophysalis granulosa

Notes: Anagnostidis and Golubic 1966

Epigloeosphaera cf. glebulenta (Zalessky) J. Komárková-Legnerová, 1991 Nomenclature:

Epigloeosphaera cf. grebulenta

Notes: Danielidis et al. 1996

Eucapsis cf. terrestris M. Akiyama, 1965

Nomenclature:

Eucapsis cf. terrestris

Notes: Lamprinou et al. 2012

Eucapsis minor (Skuja) Elenkin, 1933

Nomenclature:

Eucapsis minor

Notes: Anagnostidis and Economou-Amilli 1978

Geitlerinema acutissimum (Kufferath) Anagnostidis, 1989

Nomenclature:

Oscillatoria acutissima

Notes: Anagnostidis and Golubic 1966

Geitlerinema amphibium (C. Agardh ex Gomont) Anagnostidis, 1989

Nomenclature:

Oscillatoria amphibia f. circinata

Notes: Anagnostidis 1961

Geitlerinema apolloniae Anagnostidis, 2001

Nomenclature:

Oscillatoria splendida f. major 
Notes: Anagnostidis 1961

Geitlerinema cf. lemmermannii (Woloszynska) Anagnostidis, 1989 Nomenclature:

Oscillatoria cf. lemmermannii

Notes: Hindak and Moustaka 1988

Geitlerinema ionicum (Skuja) Anagnostidis, 1989

Nomenclature:

Oscillatoria ionica

Notes: Anagnostidis et al. 1981

Geitlerinema lemmermannii (Woloszynska) Anagnostidis, 1989

Nomenclature:

Jaaginema cf. lemmermannii

Notes: Moustaka-Gouni 1993

Geitlerinema numidicum (Gomont) Anagnostidis, 1989

Nomenclature:

Oscillatoria numidica

Notes: Anagnostidis 1961

Geitlerinema splendidum (Greville ex Gomont) Anagnostidis, 1989

Nomenclature:

Oscillatoria splendida

Notes: Anagnostidis and Golubic 1966

Geitlerinema sulphureum (Strzeszewski) Anagnostidis 2001

Nomenclature:

Oscillatoria geminata f. sulphurea

Notes: Anagnostidis 1961 
Glaucospira laxissima (G.S.West) Simic, Komárek \& Dordevic, 2014 Nomenclature:

Spirulina laxissima

Notes: Anagnostidis 1961

Gloeobacter violaceus Rippka, J. B. Waterbury \& Cohen-Bazire 1974 Nomenclature:

Aphanothece caldariorum

Notes: Anagnostidis 1961

Gloeocapsa aeruginosa Kützing, 1843

Nomenclature:

Gloeocapsa aeruginosa

Notes: Lamprinou et al. 2014

Gloeocapsa arenaria (Hassall) Rabenhorst, 1865

Nomenclature:

Gloeocapsa arenaria

Notes: Anagnostidis 1961

Gloeocapsa atrata Kützing, 1843

Nomenclature:

Gloeocapsa montana

Notes: Economou-Amilli et al. 1984

Gloeocapsa biformis Ercegovic, 1925

Nomenclature:

Gloeocapsa biformis

Notes: Anagnostidis 1968

Gloeocapsa bituminosa (Bory) Kützing, 1849

Nomenclature:

Gloeocapsa bituminosa 
Notes: Lamprinou et al. 2012

Gloeocapsa caldariorum Rabenhorst, 1865

Nomenclature:

Gloeocapsa caldariorum

Notes: Lamprinou et al. 2012

Gloeocapsa compacta Kuitzing, 1847

Nomenclature:

Gloeocapsa compacta

Notes: Anagnostidis 1968

Gloeocapsa decorticans (A. Braun) P.Richter in Wille, 1925

Nomenclature:

Gloeocapsa decorticans

Notes: Anagnostidis et al. 1983

Gloeocapsa gelatinosa Kützing, 1843

Nomenclature:

Gloeocapsa gelatinosa

Notes: Anagnostidis 1961

Gloeocapsa kuetzingiana Nägeli ex Kützing, 1849

Nomenclature:

Gloeocapsa kützingiana

Notes: Anagnostidis 1968

Gloeocapsa punctata Nägeli, 1849

Nomenclature:

Gloeocapsa punctata

Notes: Lamprinou et al. 2012 
Gloeocapsa rupestris Kützing, 1847

Nomenclature:

Gloeocapsa rupestris

Notes: Anagnostidis 1961

Gloeocapsa sanguinea (C. Agardh) Kuitzing, 1843

Nomenclature:

Gloeocapsa sanguinea

Notes: Anagnostidis 1968

Gloeocapsa thermalis Kützing, 1843

Nomenclature:

Gloeocapsa thermalis

Notes: Anagnostidis 1968

Gloeocapsa thermophila (Wood) Claus, 1959

Nomenclature:

Chroococcus thermophilus

Notes: Anagnostidis 1961

Gloeocapsopsis crepidinum (Thuret) Geitler ex Komárek, 1993

Nomenclature:

Gloeocapsopsis crepidinum

Notes: Lamprinou et al. 2012

Gloeocapsopsis cyanea (Krieger) Komárek \& Anagnostidis, 1995

Nomenclature:

Gloeocapsopsis cyanea

Notes: Lamprinou et al. 2012 
Gloeocapsopsis pleurocapsoides (Novácek) Komárek \& Anagnostidis ex Komárek, 1993

Nomenclature:

Gloeocapsopsis pleurocapsoides

Notes: Lamprinou et al. 2012

Gloeothece cf. incerta Skuja, 1964

Nomenclature:

Gloeothece cf. incerta

Notes: Lamprinou et al. 2014

Gloeothece cf. tepidariorum (A. Braun) Lagerheim 1883

Nomenclature:

Gloeothece cf. tepidariorum

Notes: Lamprinou et al. 2014

Gloeothece confluens Nägeli, 1849

Nomenclature:

Gloeothece confluens

Notes: Anagnostidis 1968

Gloeothece fusco-lutea (Nägeli ex Kützing) Nägeli, 1849

Nomenclature:

Gloeothece fusco-Iutea

Notes: Anagnostidis 1968

Gloeothece palea (Kützing) Nägeli, 1849

Nomenclature:

Gloeothece palea

Notes: Anagnostidis and Golubic 1966

Gloeothece rupestris (Lyngbye) Bornet in Wittrock \& Nordstedt, 1880 Nomenclature: 
Gloeothece rupestris

Notes: Anagnostidis 1961

Gloeothece tepidariorum (A. Braun) Lagerheim, 1883

Nomenclature:

Gloeothece rupestris var. tepidariorum

Notes: Anagnostidis 1961

Gloeotrichia echinulata P. G. Richter, 1894

Nomenclature:

Gloeotrichia echinulata

Notes: Anagnostidis 1968

Gloeotrichia pisum Thuret ex Bornet \& Flahault, 1886

Nomenclature:

Gloeotrichia pisum

Notes: Anagnostidis 1968

Gomphosphaeria aponina Kuitzing, 1836

Nomenclature:

Gomphosphaeria aponina

Notes: Anagnostidis 1968

Hapalosiphon intricatus W. \& G. S. West, 1894

Nomenclature:

Hapalosiphon intricatus

Notes: Anagnostidis 1968

Hassallia byssoidea Hassall ex Bornet \& Flahault, 1886

Nomenclature:

Tolypothrix byssoidea

Notes: Anagnostidis 1968 
Herpyzonema pulverulentum M. Hernández-Mariné \& T. Canals, 1994

Nomenclature:

Herpyzonema pulverulentum

Notes: Lamprinou et al. 2012

Heteroleibleinia infixa (Frémy) Anagnostidis \& Komárek, 1988

Nomenclature:

Lyngbya infixa

Notes: Anagnostidis 1968

Heteroleibleinia kuetzingii (Schmidle) Compère, 1985

Nomenclature:

Lyngbya kutzingii

Notes: Anagnostidis 1968

Homoeothrix caespitosa Kirchner, 1898

Nomenclature:

Homoeothrix caespitosa

Notes: Anagnostidis 1968

Homoeothrix juliana (Bornet \& Flahault ex Gomont) Kirchner, 1898

Nomenclature:

Homoeothrix juliana

Notes: Anagnostidis 1968

Hormoscilla feldmannii Anagnostidis, 2001

Nomenclature:

Borzia spongeliae

Notes: Anagnostidis 1977

Hydrococcus cesatii Rabenhorst, 1860

Nomenclature:

Hydrococcus cesatii 
Notes: Anagnostidis 1968

Hydrococcus rivularis Kützing, 1833

Nomenclature:

Hydrococcus rivularis

Notes: Anagnostidis 1968

Hydrocoleum homoeotrichum Kuitzing ex Gomont, 1892

Nomenclature:

Hydrocoleum homoeotrichum

Notes: Anagnostidis 1968

Hydrocoleum muscicola Hansgirg ex Forti, 1907

Nomenclature:

Hydrocoleum muscicolum

Notes: Anagnostidis 1968

Hydrocoleum stankovicii Čado, 1958

Nomenclature:

Hydrocoleum stankovicii

Notes: Lamprinou et al. 2012

Hyella balani Lehmann, 1903

Nomenclature:

Hyella balani

Notes: Anagnostidis and Pantazidou 1985

Hyella caespitosa Bornet \& Flahault, 1888

Nomenclature:

Hyella caespitosa

Notes: Anagnostidis and Pantazidou 1988a 
Hyella fontana Huber \& Jadin, 1892

Nomenclature:

Hyella fontana

Notes: Anagnostidis and Pantazidou 1988c

Hyella inconstans Al-Thukair \& Golubic 1991

Nomenclature:

Hyella inconstans

Notes: Pantazidou et al. 2006

Hyella kalligrammos Anagnostidis \& Pantazidou, 1988

Nomenclature:

Hyella kalligrammos

Notes: Anagnostidis and Pantazidou 1988c

Hyella maxima (Geitler) Anagnostidis \& Pantazidou, 1988

Nomenclature:

Hyella maxima

Notes: Anagnostidis and Pantazidou 1988c

Hyella reptans Al-Thukair \& Golubic, 1991

Nomenclature:

Hyella reptans

Notes: Pantazidou et al. 2006

Ifinoe spelaea Lamprinou \& Pantazidou in Lamprinou et al. 2011

Nomenclature:

Iphinoë spelaeobios

Notes: Lamprinou et al. 2011

Isactis plana Thuret ex Bornet \& Flahault, 1886

Nomenclature:

Calothrix plana 
Notes: Anagnostidis and Golubic 1966

Isocystis halobia K. Anagnostidis \& M. Roussomoustakaki, 1991

Nomenclature:

Isocystis halobia

Notes: Anagnostidis and Roussomoustakaki 1991

Isocystis pallida (Voronichin) Woronichin, 1927

Nomenclature:

Isocystis pallida

Notes: Anagnostidis 1961

Jaaginema angustissimum (W. \& G. S. West) Anagnostidis \& Komárek, 1988 Nomenclature:

Oscillatoria angustissima

Notes: Anagnostidis 1961

Jaaginema geminatum (Schwabe ex Gomont) Anagnostidis \& Komárek 1988

Nomenclature:

Oscillatoria geminata

Notes: Anagnostidis 1961

Jaaginema mauchanum (Claus) Anagnostidis \& Komárek, 1988

Nomenclature:

Oscillatoria cf. mauchiana

Notes: Lamprinou et al. 2012

Jaaginema minimum (Gicklhorn) Anagnostidis \& Komárek, 1988

Nomenclature:

Oscillatoria minima

Notes: Anagnostidis 1968 
Jaaginema profundum (Schröter \& Kirchner) Anagnostidis \& Komárek, 1988 Nomenclature:

Achroonema projundum

Notes: Anagnostidis and Economou-Amilli 1980

Jaaginema pseudogeminatum (G. Schmid) Anagnostidis \& Komárek, 1988 Nomenclature:

Oscillatoria pseudogeminata

Notes: Anagnostidis and Golubic 1966

Jaaginema subtilissimum (Kützing ex Forti) Anagnostidis \& Komárek, 1988 Nomenclature:

Oscillatoria subtilissima

Notes: Anagnostidis 1961

Johanseninema constrictum (Szafer) Hasler, Dvorák \& Poulícková, 2014 Nomenclature:

Pseudanabaena constricta

Notes: Anagnostidis and Economou-Amilli 1980

Kamptonema animale (C. Agardh ex Gomont) Strunecký, Komárek \& J. Smarda, 2014

Nomenclature:

Oscillatoria animalis

Notes: Anagnostidis 1961

Kamptonema chlorinum (Kützing ex Gomont) Strunecký, Komárek \& J. Smarda, 2014

Nomenclature:

Oscillatoria chlorina

Notes: Anagnostidis 1968 
Kamptonema cortianum (Meneghini ex Gomont) Strunecký, Komárek \& J. Smarda, 2014

Nomenclature:

Oscillatoria cortiana

Notes: Anagnostidis 1961

Kamptonema formosum (Bory ex Gomont) Strunecký, Komárek \& J. Smarda, 2014

Nomenclature:

Oscillatoria formosa

Notes: Anagnostidis 1961

Kamptonema jasorvense (Vouk) Strunecký, Komárek \& J. Smarda, 2014

Nomenclature:

Oscillatoria jasorvensis

Notes: Anagnostidis and Golubic 1966

Kamptonema laetevirens (H.M.Crouan \& P.L.Crouan ex Gomont) Strunecký, Komárek \& J. Smarda, 2014

Nomenclature:

Phormidium laetevirens

Notes: Anagnostidis and Roussomoustakaki 1988

Kamptonema okenii (C.Agardh ex Gomont) Strunecký, Komárek \& J. Smarda, 2014

Nomenclature:

Oscillatoria okenii

Notes: Anagnostidis 1961

Komvophoron cf. crassum (Vozzennikova) Anagnostidis \& Komárek, 1988

Nomenclature:

Komvophoron cf. crassum

Notes: Anagnostidis and Roussomoustakaki 1991 
Komvophoron pallidum (Skuja) Anagnostidis \& Komárek, 1988

Nomenclature:

Pseudanabaena pallida

Notes: Anagnostidis and Economou-Amilli 1980

Komvophoron schmidlei (Jaag) Anagnostidis \& Komárek, 1988

Nomenclature:

Pseudanabaena schmidlei

Notes: Anagnostidis 1968

Kyrtuthrix dalmatica Ercegović, 1929

Nomenclature:

Kyrtuthrix dalmatica

Notes: Anagnostidis 1968

Leibleinia gracilis (Rabenhorst ex Gomont) Anagnostidis \& Komárek, 1988

Nomenclature:

Lyngbya gracilis

Notes: Anagnostidis and Golubic 1966

Leibleinia nordgaardii Anagnostidis \& Komárek, 1988

Nomenclature:

Lyngbya nordagardhii

Notes: Anagnostidis 1968

Lemmermanniella pallida (Lemmermann) Geitler, 1942

Nomenclature:

Lemmermanniella pallida

Notes: Tryfon et al. 1997 
Leptolyngbya amplivaginata (van Goor) Anagnostidis \& Komárek, 1988

Nomenclature:

Lyngbya amplivaginata

Notes: Anagnostidis 1968

Leptolyngbya angustissima (W. \& G. S. West) Anagnostidis \& Komárek, 1988 Nomenclature:

Phormidium angustissimum

Notes: Anagnostidis 1961

Leptolyngbya antarctica (W. \& G. S. West) Anagnostidis \& Komárek, 1988

Nomenclature:

Phormidium antarcticum

Notes: Anagnostidis et al. 1982

Leptolyngbya boryana (Gomont) Anagnostidis \& Komárek, 1988

Nomenclature:

Plectonema boryanum

Notes: Anagnostidis et al. 1981

Leptolyngbya calotrichoides (Gomont) Anagnostidis \& Komárek, 1988

Nomenclature:

Plectonema calotrichoides

Notes: Anagnostidis 1968

Leptolyngbya cebennensis (Gomont) I. Umezaki \& M. Watanabe, 1994

Nomenclature:

Leptolyngbya cebennensis

Notes: Lamprinou et al. 2012 
Leptolyngbya cf. carnea (Kützing ex Lemmermann) Anagnostidis \& Komárek, 1988

Nomenclature:

Leptolyngbya cf. carnea

Notes: Lamprinou et al. 2012

Leptolyngbya cf. laminosa (Gomont ex Gomont) Anagnostidis \& Komárek, 1988 Nomenclature:

Leptolyngbya cf. laminosa

Notes: Lamprinou et al. 2014

Leptolyngbya compacta (Kützing ex Hansgirg) Komárek in Anagnostidis, 2001

Nomenclature:

Leptolyngbya compacta

Notes: Lamprinou et al. 2012

Leptolyngbya ectocarpi (Gomont) Anagnostidis \& Komárek, 1988

Nomenclature:

Phormidium ectocarpi

Notes: Anagnostidis 1968

Leptolyngbya ercegovicii (Čado) Anagnostidis \& Komárek, 1988

Nomenclature:

Leptolyngbya ercegovicii

Notes: Lamprinou et al. 2012

Leptolyngbya foveolara (Gomont) Anagnostidis \& Komárek, 1988

Nomenclature:

Phormidium foveolarum

Notes: Anagnostidis 1961 
Leptolyngbya fragilis (Gomont) Anagnostidis \& Komárek, 1988

Nomenclature:

Phormidium fragile

Notes: Anagnostidis 1961

Leptolyngbya gracillima (Hansgirg) Anagnostidis \& Komárek, 1988

Nomenclature:

Plectonema gracillimum

Notes: Anagnostidis et al. 1982

Leptolyngbya halophila (Hansgirg ex Gomont) Anagnostidis \& Komárek, 1988

Nomenclature:

Lyngbya halophila

Notes: Anagnostidis 1968

Leptolyngbya lagerheimii (Gomont ex Gomont) Anagnostidis \& Komárek, 1988 Nomenclature:

Lyngbya lagerheimii

Notes: Anagnostidis 1961

Leptolyngbya laminosa (Gomont ex Gomont) Anagnostidis \& Komárek, 1988

Nomenclature:

Phormidium laminosum

Notes: Anagnostidis 1961

Leptolyngbya lurida (Gomont) Anagnostidis \& Komárek, 1988

Nomenclature:

Phormidium luridum

Notes: Anagnostidis 1961

Leptolyngbya nana (Tilden) Anagnostidis \& Komárek, 1988

Nomenclature:

Leptolyngbya nana 
Notes: Lamprinou et al. 2012

Leptolyngbya norvegica (Gomont) Anagnostidis \& Komárek, 1988

Nomenclature:

Plectonema norvegicum

Notes: Anagnostidis 1968

Leptolyngbya nostocorum (Bornet ex Gomont) Anagnostidis \& Komárek, 1988

Nomenclature:

Plectonema nostocorum

Notes: Anagnostidis and Golubic 1966

Leptolyngbya notata (Schmidle) Anagnostidis \& Komárek, 1988

Nomenclature:

Plectonema notatum

Notes: Anagnostidis 1968

Leptolyngbya ochracea (Thuret ex Gomont) Anagnostidis \& Komárek 1988

Nomenclature:

Lyngbya ochracea

Notes: Anagnostidis 1968

Leptolyngbya perelegans (Lemmermann) Anagnostidis \& Komárek, 1988

Nomenclature:

Lyngbya perelegans

Notes: Anagnostidis and Golubic 1966

Leptolyngbya perforans (Geitler) Anagnostidis \& Komárek, 1988

Nomenclature:

Schizothrix perforans st. typicus

Notes: Anagnostidis 1968 
Leptolyngbya phormidioides (Anagnostidis) Anagnostidis \& Komárek, 1988

Nomenclature:

Oscillatoria acuminata f. phormidioides

Notes: Anagnostidis 1961

Leptolyngbya purpurascens (Gomont ex Gomont) Anagnostidis \& Komárek, 1988

Nomenclature:

Leptolyngbya purpurascens

Notes: Lamprinou et al. 2012

Leptolyngbya rivulariarum (Gomont) Anagnostidis \& Komárek 1988

Nomenclature:

Lyngbya rivulariarum

Notes: Anagnostidis 1968

Leptolyngbya subtilissima (Hansgirg) Komárek in Anagnostidis, 2001 Nomenclature:

Leptolyngbya subtilissima

Notes: Lamprinou et al. 2014

Leptolyngbya subuliformis (Gomont) Anagnostidis \& Komárek, 1988

Nomenclature:

Phormidium subuliforme

Notes: Anagnostidis 1961

Leptolyngbya tenuis (Gomont) Anagnostidis \& Komárek, 1988

Nomenclature:

Phormidium tenue f. circinata

Notes: Anagnostidis 1961 
Leptolyngbya terebrans (Bornet \& Flahault ex Gomont) Anagnostidis \& Komárek, 1988

Nomenclature:

Plectonema terebrans

Notes: Anagnostidis and Golubic 1966

Leptolyngbya truncata (Lemmermann) Anagnostidis \& Komárek, 1988

Nomenclature:

Phormidium truncatum

Notes: Anagnostidis 1961

Leptolyngbya undosa (Čado) Anagnostidis \& Komárek, 1988

Nomenclature:

Leptolyngbya undosa

Notes: Lamprinou et al. 2012

Leptolyngbya valderiana (Gomont) Anagnostidis \& Komárek 1988

Nomenclature:

Phormidium valderianum

Notes: Anagnostidis 1961

Leptolyngbya weedii (Drouet) Anagnostidis 2001

Nomenclature:

Phormidium weedii

Notes: Anagnostidis and Economou-Amilli 1978

Limnococcus limneticus (Lemmermann) Komárková, Jezberová, O. Komárek \& Zapomelová, 2010

Nomenclature:

Chroococcus limneticus

Notes: Anagnostidis 1968 
Limnothrix brachynema (Skuja) Hindák \& Trifonova, 1989

Nomenclature:

Lyngbya brachynema

Notes: Anagnostidis 1968

Limnothrix obliqueacuminata (Skuja) Meffert 1988

Nomenclature:

Oscillatoria obliqueacuminata

Notes: Anagnostidis 1968

Limnothrix redekei (Goor) Meffert, 1988

Nomenclature:

Limnothrix redekei

Notes: Vardaka et al. 2000

Lyngbya f. minor Elenkin, 1949

Nomenclature:

Lyngbya aerugineo-coerulea f. minor

Notes: Anagnostidis 1961

Lyngbya aestuarii Liebman ex Gomont, 1892

Nomenclature:

Lyngbya aestuarii

Notes: Anagnostidis 1968

Lyngbya agardhii Gomont, 1892

Nomenclature:

Lyngbya agardhii

Notes: Anagnostidis and Golubic 1966

Lyngbya boryana Kirchner ex Hansgirg, 1892

Nomenclature:

Phormidium boryanum 
Notes: Anagnostidis 1961

Lyngbya cf. spiralis Geitler, 1932

Nomenclature:

Lyngbya cf. spiralis

Notes: Overbeck et al. 1982

Lyngbya confervoides C. Agardh ex Gomont, 1892

Nomenclature:

Lyngbya confervoides

Notes: Anagnostidis and Golubic 1966

Lyngbya lutea Gomont ex Gomont, 1892

Nomenclature:

Lyngbya lutea

Notes: Anagnostidis and Golubic 1966

Lyngbya majuscula Harvey ex Gomont, 1892

Nomenclature:

Lyngbya majuscula

Notes: Anagnostidis 1968

Lyngbya martensiana Meneghini ex Gomont, 1892

Nomenclature:

Lyngbya martensiana

Notes: Anagnostidis 1961

Lyngbya palikiana Claus, 1955

Nomenclature:

Leptolyngbya palikiana

Notes: Lamprinou et al. 2012 
Lyngbya semiplena J. Agardh ex Gomont, 1892

Nomenclature:

Lyngbya semiplena

Notes: Anagnostidis and Golubic 1966

Lyngbya sordida Gomont, 1892

Nomenclature:

Lyngbya sordida

Notes: Anagnostidis and Golubic 1966

Mastigocladus laminosus Cohn ex Kirchner, 1898

Nomenclature:

Mastigocladus laminosus

Notes: Anagnostidis 1961

Mastigocoleus testarum Lagerheim ex Bornet \& Flahault, 1887

Nomenclature:

Mastigocoleus testarum

Notes: Anagnostidis 1968

Merismopedia elegans A. Braun ex Kuitzing, 1849

Nomenclature:

Merismopedia elegans

Notes: Anagnostidis 1968

Merismopedia glauca (Ehrenberg) Kützing, 1845

Nomenclature:

Merismopedia glauca

Notes: Anagnostidis 1968

Merismopedia mediterranea Nägeli, 1849

Nomenclature:

Merismopedia glauca f. mediterranea 
Notes: Anagnostidis and Golubic 1966

Merismopedia minima G. Beck 1897

Nomenclature:

Merismopedia minima

Notes: Anagnostidis 1968

Merismopedia punctata Meyen, 1839

Nomenclature:

Merismopedia punctata

Notes: Anagnostidis 1968

Merismopedia smithii De Toni, 1939

Nomenclature:

Merismopedia maior

Notes: Anagnostidis 1968

Merismopedia tenuissima Lemmermann 1898

Nomenclature:

Merismopedia tenuissima

Notes: Anagnostidis 1968

Merismopedia trolleri Bachmann, 1920

Nomenclature:

Merismopedia trolleri

Notes: Anagnostidis 1968

Merismopedia warmingiana (Lagerheim) Forti, 1883

Nomenclature:

Merismopedia warmingiana

Notes: Anagnostidis 1968 
Microchaete grisea Thuret ex Bornet \& Flahault, 1886

Nomenclature:

Microchaete grisea

Notes: Anagnostidis 1968

Microchaete tenera Thuret ex Bornet \& Flahault, 1886

Nomenclature:

Microchaete tenera

Notes: Anagnostidis 1968

Microcoleus amoenus (Gomont) Strunecky, Komárek \& J. R. Johansen, 2013 Nomenclature:

Oscillatoria amoena

Notes: Anagnostidis 1968

Microcoleus amoenus (Gomont) Strunecky, Komárek \& J. R. Johansen, 2013 Nomenclature:

Phormidium amoenum

Notes: Anagnostidis and Roussomoustakaki 1991

Microcoleus autumnalis (Gomont) Strunecky, Komárek \& J. R. Johansen, 2013 Nomenclature:

Phormidium autumnale

Notes: Anagnostidis 1961

Microcoleus ferrugineus Frémy, 1936

Nomenclature:

Microcoleus ferrugineus

Notes: Anagnostidis 1968

Microcoleus lacustris Farlow ex Gomont, 1892

Nomenclature:

Microcoleus lacustris 
Notes: Anagnostidis 1968

Microcoleus paludosus Gomont, 1892

Nomenclature:

Microcoleus paludosus

Notes: Anagnostidis 1968

Microcoleus setchellianus (Gomont) Strunecky, Komárek \& J. R. Johansen, 2013

Nomenclature:

Phormidium setchellianum

Notes: Lamprinou et al. 2012

Microcoleus steenstrupii J. B. Petersen, 1923

Nomenclature:

Microcoleus steenstrupii

Notes: Lamprinou et al. 2012

Microcoleus vaginatus Gomont ex Gomont, 1892

Nomenclature:

Microcoleus vaginatus

Notes: Anagnostidis 1968

Microcoleus vulgaris Strunecky, Komárek \& J. R. Johansen 2013

Nomenclature:

Phormidium vulgare

Notes: Lamprinou et al. 2012

Microcystis flos-aquae (Wittrock) Kirchner, 1898

Nomenclature:

Microcystis flos-aquae

Notes: Anagnostidis 1961 
Microcystis aeruginosa (Kützing) Kützing, 1846

Nomenclature:

Microcystis aeruginosa

Notes: Anagnostidis 1968

Microcystis ichthyoblabe (G.Kunze) Kützing, 1846

Nomenclature:

Microcystis ichthyoblabe

Notes: Tryfon et al. 1997

Microcystis incerta (Lemmermann) Lemmermann, 1903

Nomenclature:

Microcystis incerta

Notes: Overbeck et al. 1982

Microcystis marginata (Meneghini) Kützing, 1846

Nomenclature:

Microcystis marginata

Notes: Anagnostidis 1968

Microcystis novacekii (Komárek) Compère, 1974

Nomenclature:

Microcystis novacekii

Notes: Vardaka et al. 2000

Microcystis panniformis Komárek, Komárková-Legnerová, Sant'Anna, M. T. P. Azevedo, \& P. A. C. Senna, 2002

Nomenclature:

Microcystis panniformis

Notes: Papadimitriou et al. 2013 
Microcystis pulverea (H. C. Wood) Forti, 1907

Nomenclature:

Microcystis pulverea

Notes: Anagnostidis 1968

Microcystis smithii Komárek \& Anagnostidis, 1995

Nomenclature:

Aphanocapsa pulchra

Notes: Anagnostidis 1968

Microcystis viridis (A. Braun) Lemmermann, 1903

Nomenclature:

Microcystis viridis

Notes: Anagnostidis 1968

Microcystis wesenbergii (Komárek) Komárek ex Komárek, 1968

Nomenclature:

Microcystis wesenbergii

Notes: Tryfon et al. 1996

Myxosarcina concinna Printz, 1921

Nomenclature:

Myxosarcina concinna

Notes: Anagnostidis et al. 1983

Nodularia harveyana Thuret ex Bornet \& Flahault, 1886

Nomenclature:

Nodularia harveyana

Notes: Anagnostidis 1968

Nodularia spumigena Mertens ex Bornet \& Flahault 1888

Nomenclature:

Nodularia spumigena 
Notes: Anagnostidis 1968

Nostoc calcicola Brébisson ex Bornet \& Flahault, 1886

Nomenclature:

Nostoc calcicola

Notes: Anagnostidis 1961

Nostoc commune Vaucher ex Bornet \& Flahault, 1888

Nomenclature:

Nostoc commune

Notes: Economou-Amilli et al. 1984

Nostoc humifusum Carmichael ex Bornet \& Flahault, 1888

Nomenclature:

Nostoc humifusum

Notes: Anagnostidis 1961

Nostoc letestui Frémy, 1930

Nomenclature:

Nostoc letestui

Notes: Lamprinou et al. 2012

Nostoc linckia Bornet ex Bornet \& Flahault, 1888

Nomenclature:

Nostoc linckia

Notes: Anagnostidis 1968

Nostoc microscopicum Carmichael ex Bornet \& Flahault, 1888

Nomenclature:

Nostoc microscopicum

Notes: Anagnostidis 1968 
Nostoc minutissimum Kützing ex Bornet \& Flahault, 1888

Nomenclature:

Nostoc minutissimum

Notes: Anagnostidis 1968

Nostoc paludosum Kützing ex Bornet \& Flahault, 1888

Nomenclature:

Nostoc paludosum

Notes: Anagnostidis 1961

Nostoc punctiforme Hariot 1891

Nomenclature:

Nostoc punctiforme

Notes: Anagnostidis et al. 1981

Nostoc sphaericum Vaucher ex Bornet \& Flahault, 1888

Nomenclature:

Nostoc sphaericum

Notes: Anagnostidis 1968

Oculatella subterranea Zammit, Billi \& Albertano, 2012

Nomenclature:

Oculatella subterranea

Notes: Christodoulou et al. 2015

Oscillatoria anguina Bory ex Gomont 1892

Nomenclature:

Oscillatoria anguina

Notes: Anagnostidis 1961

Oscillatoria bonnemaisonii P. Crouan \& H. Crouan ex Gomont, 1892

Nomenclature:

Oscillatoria bonnemaisonii 
Notes: Anagnostidis 1968

Oscillatoria corallinae Gomont ex Gomont, 1892

Nomenclature:

Oscillatoria corallinae

Notes: Anagnostidis and Golubic 1966

Oscillatoria crassa (C. B. Rao) Anagnostidis, 2001

Nomenclature:

Oscillatoria ornata var. crassa

Notes: Anagnostidis 1961

Oscillatoria curviceps C. Agardh ex Gomont, 1892

Nomenclature:

Oscillatoria curviceps

Notes: Anagnostidis 1968

Oscillatoria laetevirens Hofman-Bang ex Forti 1892

Nomenclature:

Oscillatoria laetevirens

Notes: Anagnostidis 1961

Oscillatoria limosa C. Agardh ex Gomont 1892

Nomenclature:

Oscillatoria limosa

Notes: Anagnostidis and Golubic 1966

Oscillatoria margaritifera Kützing ex Gomont, 1892

Nomenclature:

Oscillatoria margaritifera

Notes: Anagnostidis 1968 
Oscillatoria okenii var. gracilis (Kützing) Kützing ex Forti, 1904 Nomenclature:

Oscillatoria okenii f. gracilis

Notes: Anagnostidis 1961

Oscillatoria ornata Kützing ex Gomont, 1892

Nomenclature:

Oscillatoria ornata

Notes: Anagnostidis 1961

Oscillatoria princeps Vaucher ex Gomont, 1892

Nomenclature:

Oscillatoria princeps

Notes: Anagnostidis 1961

Oscillatoria pseudoangusta Claus, 1955

Nomenclature:

Oscillatoria pseudoangusta

Notes: Anagnostidis 1961

Oscillatoria putrida Schmidle, 1901

Nomenclature:

Oscillatoria putrida

Notes: Anagnostidis 1968

Oscillatoria rhamphoidea Anagnostidis, 2001

Nomenclature:

Oscillatoria salina f. major

Notes: Anagnostidis et al. 1981

Oscillatoria rupicola (Hansgirg) Hansgirg ex Forti, 1907

Nomenclature:

Oscillatoria rupicola 
Notes: Lamprinou et al. 2012

Oscillatoria sancta Kützing ex Gomont 1892

Nomenclature:

Oscillatoria sancta

Notes: Anagnostidis 1961

Oscillatoria simplicissima Gomont, 1892

Nomenclature:

Oscillatoria simplicissima

Notes: Anagnostidis 1961

Oscillatoria subbrevis Schmidle, 1901

Nomenclature:

Oscillatoria subbrevis

Notes: Anagnostidis et al. 1981

Oscillatoria tenuis C. Agardh ex Gomont, 1892

Nomenclature:

Oscillatoria tenuis

Notes: Anagnostidis 1961

Oscillatoria var. phormidioides Hansgirg ex Drouet, 1957

Nomenclature:

Oscillatoria okenii f. phormidioides

Notes: Anagnostidis 1961

Oscillatoria trichoides Szafer, 1910

Nomenclature:

Oscillatoria trichoides

Notes: Anagnostidis and Golubic 1966 
Oxynema acuminatum (Gomont) Chatchawan, Komárek, Strunecky, Smarda \& Peerapornpisal, 2012

Nomenclature:

Oscillatoria acuminata

Notes: Anagnostidis 1961

Pannus spumosus B.Hickel, 1991

Nomenclature:

Pannus spumosus

Notes: Hindak and Moustaka 1988

Petalonema densum (Bornet ex Bornet \& Flahault) Migula, 1907

Nomenclature:

Petalonema densum

Notes: Anagnostidis 1968

Phormidesmis molle (Gomont) Turicchia, Ventura, Komárková \& Komárek, 2009

Nomenclature:

Phormidium molle

Notes: Anagnostidis 1961

Phormidiochaete nordstedtii (Bornet et Flahault) Komárek in Anagnostidis, 2001

Nomenclature:

Phormidiochaete nordstedtii

Notes: Lamprinou et al. 2012

Phormidium acuminatum var. longe-attenuatum (Geitler \& Ruttner) I. Umezaki \& M. Watanabe, 1994

Nomenclature:

Oscillatoria acuminata f. longe-attenuata

Notes: Anagnostidis 1961 
Phormidium aerugineo-caeruleum (Gomont) Anagnostidis \& Komárek, 1988

Nomenclature:

Lyngbya aerugineo-coerulea

Notes: Anagnostidis 1961

Phormidium ambiguum Gomont, 1892

Nomenclature:

Phormidium ambiguum

Notes: Anagnostidis 1961

Phormidium articulatum (N. L. Gardner) Anagnostidis \& Komárek, 1988

Nomenclature:

Phormidium articulatum

Notes: Lamprinou et al. 2012

Phormidium boryanum Kützing, 1843

Nomenclature:

Oscillatoria boryana

Notes: Anagnostidis and Golubic 1966

Phormidium breve (Kützing ex Gomont) Anagnostidis \& Komárek, 1988

Nomenclature:

Oscillatoria brevis

Notes: Anagnostidis 1961

Phormidium cf. griseoviolaceum (Skuja) Anagnostidis, 2001

Nomenclature:

Phormidium cf. griseoviolaceum

Notes: Lamprinou et al. 2012

Phormidium chalybeum (Mertens ex Gomont) Anagnostidis \& Komárek, 1988 Nomenclature:

Oscillatoria chalybea 
Notes: Anagnostidis 1961

Phormidium corium Gomont ex Gomont, 1892

Nomenclature:

Phormidium corium

Notes: Anagnostidis 1961

Phormidium diguetii (Gomont) Anagnostidis \& Komárek, 1988

Nomenclature:

Lyngbya diguetii

Notes: Anagnostidis 1961

Phormidium edessae Skuja, 1937

Nomenclature:

Phormidium edessae

Notes: Anagnostidis 1968

Phormidium endophyticum (W. \& G. S. West) Skuja, 1956

Nomenclature:

Phormidium endophyticum

Notes: Anagnostidis 1968

Phormidium favosum Gomont, 1892

Nomenclature:

Phormidium favosum

Notes: Anagnostidis 1968

Phormidium feldmanni Frémy, 1937

Nomenclature:

Phormidium feldmanni

Notes: Anagnostidis 1968 
Phormidium grunowianum (Gomont) Anagnostidis \& Komárek, 1988

Nomenclature:

Oscillatoria grunowiana

Notes: Anagnostidis 1961

Phormidium henningsii (Lemmermann) Anagnostidis, 2001

Nomenclature:

Leptolyngbya henningsii

Notes: Lamprinou et al. 2012

Phormidium incrustatum Gomont ex Gomont, 1892

Nomenclature:

Phormidium incrustans

Notes: Anagnostidis 1968

Phormidium interruptum Kützing ex Gomont, 1892

Nomenclature:

Phormidium interruptum

Notes: Lamprinou et al. 2012

Phormidium inundatum Kuitzing ex Gomont, 1892

Nomenclature:

Phormidium inundatum

Notes: Anagnostidis 1961

Phormidium janthiphorum Elenkin, 1949

Nomenclature:

Oscillatoria janthiphora

Notes: Anagnostidis and Golubic 1966

Phormidium jenkelianum Kützing ex Gomont, 1892

Nomenclature:

Phormidium jenkelianum 
Notes: Anagnostidis 1961

Phormidium kuetzingianum (Kirchner ex Gomont) Anagnostidis \& Komárek, 1988

Nomenclature:

Phormidium kuetzingianum

Notes: Lamprinou et al. 2012

Phormidium lacustre (Čado) Anagnostidis, 2001

Nomenclature:

Phormidium lacustre

Notes: Lamprinou et al. 2012

Phormidium litorale Golubic, 1960

Nomenclature:

Phormidium litorale

Notes: Anagnostidis and Golubic 1966

Phormidium macedonicum (Čado), 1959

Nomenclature:

Phormidium macedonicum

Notes: Lamprinou et al. 2012

Phormidium melanochroun Lamprinou \& Pantazidou, 2013

Nomenclature:

Phormidium melanochroun

Notes: Lamprinou et al. 2013b

Phormidium minutum Lindstedt, 1943

Nomenclature:

Phormidium minutum

Notes: Anagnostidis 1968 
Phormidium molischii (Vouk) Anagnostidis \& Komárek, 1988

Nomenclature:

Lyngbya molischi

Notes: Anagnostidis 1968

Phormidium molle var. tenuior W. \& G. S. West ex Geitler, 1925

Nomenclature:

Phormidium molle f. tenuior

Notes: Anagnostidis 1961

Phormidium nigroviride (Thwaites ex Gomont) Anagnostidis \& Komárek, 1988

Nomenclature:

Oscillatoria nigroviridis

Notes: Anagnostidis and Golubic 1966

Phormidium nigrum (Vaucher ex Gomont) Anagnostidis \& Komárek, 1988

Nomenclature:

Oscillatoria nigra

Notes: Anagnostidis 1968

Phormidium pachydermaticum Frémy, 1930

Nomenclature:

Phormidium pachydermaticum

Notes: Anagnostidis 1961

Phormidium papyraceum Gomont ex Gomont, 1892

Nomenclature:

Phormidium papyraceum

Notes: Anagnostidis 1961

Phormidium priestleyi F. E. Fritsch, 1917

Nomenclature:

Phormidium priestleyi 
Notes: Lamprinou et al. 2012

Phormidium retzii Kützing ex Gomont, 1892

Nomenclature:

Phormidium retzii

Notes: Anagnostidis 1961

Phormidium subfuscum Kützing ex Gomont. 1892

Nomenclature:

Phormidium subfuscum

Notes: Anagnostidis et al. 1981

Phormidium submembranaceum Gomont, 1892

Nomenclature:

Phormidium submembranaceum

Notes: Anagnostidis 1968

Phormidium tanganyikae (G. S. West) Anagnostidis \& Komárek, 1988

Nomenclature:

Oscillatoria tanganyikae

Notes: Anagnostidis et al. 1981

Phormidium terebriforme (C. Agardh ex Gomont) Anagnostidis \& Komárek, 1988

Nomenclature:

Oscillatoria terebriformis

Notes: Anagnostidis 1961

Phormidium tergestinum Kuitzing ex Anagnostidis \& Komárek, 1988

Nomenclature:

Phormidium tergestinum

Notes: Lamprinou et al. 2012 
Phormidium thwaitesii I. Umezaki \& M. Watanabe, 1994

Nomenclature:

Oscillatoria subuliformis

Notes: Anagnostidis 1968

Phormidium uncinatum Gomont ex Gomont, 1892

Nomenclature:

Phormidium uncinatum

Notes: Anagnostidis 1968

Phormidium willei (N. L. Gardner) Anagnostidis \& Komárek, 1988

Nomenclature:

Oscillatoria willei

Notes: Anagnostidis and Golubic 1966

Planktolyngbya bipunctata (Lemmermann) Anagnostidis \& Komárek, 1988 Nomenclature:

Lyngbya bipunctata

Notes: Anagnostidis and Golubic 1966

Planktolyngbya circumcreta (G. S. West) Anagnostidis \& Komárek, 1988 Nomenclature:

Lyngbya circumcreta

Notes: Moustaka-Gouni 1988

Planktolyngbya contorta (Lemmermann) Anagnostidis \& Komárek, 1988 Nomenclature:

Lyngbya contorta

Notes: Anagnostidis 1968 
Planktolyngbya limnetica (Lemmermann) Komárková- Legnerová \& Cronberg, 1992

Nomenclature:

Lyngbya limnetica

Notes: Anagnostidis 1961

Planktothrix agardhii (Gomont) Anagnostidis \& Komárek, 1988

Nomenclature:

Planktothrix agardhii

Notes: Papadimitriou et al. 2013

Planktothrix isothrix (Skuja) Komárek \& Komárková, 2004

Nomenclature:

Oscillatoria cf. agardhii var. isothrix

Notes: Overbeck et al. 1982

Planktothrix mougeotii Anagnostidis \& Komárek, 1988

Nomenclature:

Planktothrix mougeotii

Notes: Anagnostidis and Pantazidou 1988c

Planktothrix rubescens (De Candolle ex Gomont) Anagnostidis \& Komárek, 1988

Nomenclature:

Planktothrix rubescens

Notes: Vareli et al. 2009

Plectonema araucanum Schwabe, 1960

Nomenclature:

Plectonema araucanum

Notes: Lamprinou et al. 2012 
Plectonema tomasinianum Bornet ex Gomont, 1893

Nomenclature:

Plectonema tomassinianum

Notes: Anagnostidis and Economou-Amilli 1978

Pleurocapsa fuliginosa Hauck, 1885

Nomenclature:

Pleurocapsa fuliginosa

Notes: Lamprinou et al. 2012

Pleurocapsa minor Hansgirg, 1891

Nomenclature:

Scopulonema minus

Notes: Anagnostidis 1968

Porphyrosiphon versicolor (Gomont) Anagnostidis \& Komárek, 1988

Nomenclature:

Lyngbya versicolor

Notes: Anagnostidis 1968

Pseudanabaena amphigranulata (Van Goor) Anagnostidis, 2001

Nomenclature:

Pseudanabaena galeata f. tenuis

Notes: Anagnostidis 1961

Pseudanabaena arcuata (Skuja) Anagnostidis \& Komárek, 1988

Nomenclature:

Phormidium arcuatum

Notes: Anagnostidis 1968

Pseudanabaena biceps Böcher, 1946

Nomenclature:

Pseudanabaena biceps 
Notes: Anagnostidis 1968

Pseudanabaena catenata Lauterborn, 1915

Nomenclature:

Pseudanabaena catenata

Notes: Anagnostidis 1961

Pseudanabaena galeata Böcher, 1949

Nomenclature:

Pseudanabaena galeata

Notes: Anagnostidis 1961

Pseudanabaena limnetica (Lemmermann) Komárek, 1974

Nomenclature:

Oscillatoria limnetica

Notes: Anagnostidis 1968

Pseudanabaena lonchoides Anagnostidis, 1961

Nomenclature:

Pseudanabaena lonchoides

Notes: Anagnostidis 1961

Pseudanabaena mucicola (Naumann \& Huber-Pestalozzi) Schwabe, 1964 Nomenclature:

Phormidium mucicola

Notes: Anagnostidis 1968

Pseudanabaena raphidioides (Geitler) Anagnostidis \& Komárek, 1988 Nomenclature:

Pseudanabaena raphidioides

Notes: Montesanto et al. 1999 
Pseudocapsa dubia Ercegovic, 1925

Nomenclature:

Pseudocapsa dubia

Notes: Anagnostidis and Pantazidou 1991a

Pseudophormidium battersii (Gomont) Anagnostidis, 2001

Nomenclature:

Plectonema battersii

Notes: Anagnostidis 1968

Pseudophormidium golenkinianum (Gomont) Anagnostidis, 2001

Nomenclature:

Plectonema golenkinianum

Notes: Anagnostidis and Golubic 1966

Pseudophormidium hollerbachianum (Elenkin) Anagnostidis, 2001

Nomenclature:

Plectonema boryanum f. hollerbachianum

Notes: Anagnostidis et al. 1983

Pseudophormidium radiosum (Gomont) Anagnostidis \& Komárek, 1988

Nomenclature:

Plectonema radiosum

Notes: Anagnostidis 1968

Pseudophormidium spelaeoides (Čado) Anagnostidis, 2001

Nomenclature:

Pseudophormidium spelaeoides

Notes: Lamprinou et al. 2012

Pseudophormidium tenue (Thuret ex Gomont) Anagnostidis \& Komárek, 1988 Nomenclature:

Plectonema tenue 
Notes: Anagnostidis and Economou-Amilli 1978

Pseudoscytonema endolithicum (Ercegovic) Anagnostidis in Komárek \& Anagnostidis, 2005

Nomenclature:

Plectonema endolithicum

Notes: Anagnostidis 1968

Radiocystis geminata Skuja, 1948

Nomenclature:

Radiocystis geminata

Notes: Anagnostidis 1968

Raphidiopsis mediterranea Skuja, 1937

Nomenclature:

Raphidiopsis mediterranea

Notes: Anagnostidis 1968

Rhabdoderma curtum (Setchell) Komárek \& Anagnostidis, 1995

Nomenclature:

Synechococcus curtus

Notes: Anagnostidis and Golubic 1966

Rhabdoderma lineare Schmidle \& Lauterborn in Schmidle, 1900

Nomenclature:

Synechococcus linearis

Notes: Moustaka 1988

Rhabdogloea cf. elenkinii (Roll) Komárek \& Anagnostidis, 1995

Nomenclature:

Dactylococcopsis cf. elenkinii

Notes: Hindak and Moustaka 1988 
Rivularia atra Roth ex Bornet \& Flahault, 1886

Nomenclature:

Rivularia atra

Notes: Anagnostidis 1968

Rivularia biasolettiana Meneghini ex Bornet \& Flahault, 1886

Nomenclature:

Rivularia biasoletiana

Notes: Anagnostidis 1968

Rivularia bullata Berkeley ex Bornet \& Flahault, 1886

Nomenclature:

Rivularia bullata

Notes: Anagnostidis 1968

Rivularia calcarata (Woronichin) Poljankij in Hollerbach et al. 1953 Nomenclature:

Dichothrix compacta var. calcarata

Notes: Anagnostidis 1968

Rivularia haematites C. Agardh ex Bornet \& Flahault, 1886

Nomenclature:

Rivularia haematites

Notes: Anagnostidis 1968

Rivularia nitida C. Agardh ex Bornet \& Flahault, 1886

Nomenclature:

Rivularia nitida

Notes: Anagnostidis 1968

Rivularia polyotis Roth ex Bornet \& Flahault, 1886

Nomenclature:

Rivularia polyotis 
Notes: Anagnostidis and Golubic 1966

Romeria gracilis (Koczwara) Koczwara in Geitler, 1932

Nomenclature:

Romeria gracilis

Notes: Tryfon et al. 1996

Romeria simplex (Hindák) Hindák, 1988

Nomenclature:

Romeria simplex

Notes: Katsiapi et al. 2012

Schizothrix affinis Lemmermann, 1905

Nomenclature:

Schizothrix affinis

Notes: Anagnostidis 1968

Schizothrix arenaria Gomont, 1892

Nomenclature:

Schizothrix arenaria

Notes: Anagnostidis 1968

Schizothrix bosniaca (Forti) Geitler, 1932

Nomenclature:

Schizothrix bosniaca

Notes: Anagnostidis et al. 1983

Schizothrix calcicola Gomont, 1892

Nomenclature:

Schizothrix calcicola

Notes: Anagnostidis 1968 
Schizothrix coriacea Gomont, 1892

Nomenclature:

Schizothrix coriacea

Notes: Anagnostidis 1968

Schizothrix delicatissima W. \& G. S. West, 1897

Nomenclature:

Schizothrix delicatissima

Notes: Anagnostidis 1968

Schizothrix fasciculata Gomont ex Gomont, 1892

Nomenclature:

Schizothrix fasciculata

Notes: Anagnostidis 1968

Schizothrix heufleri Grunow ex Gomont, 1892

Nomenclature:

Schizothrix heufleri

Notes: Anagnostidis 1968

Schizothrix lacustris A. Braun ex Gomont, 1892

Nomenclature:

Schizothrix lacustris

Notes: Anagnostidis 1968

Schizothrix lardacea Gomont, 1892

Nomenclature:

Schizothrix lardacea

Notes: Anagnostidis 1968

Schizothrix lateritia Gomont, 1892

Nomenclature:

Schizothrix lateritia 
Notes: Anagnostidis 1968

Schizothrix lenormandiana Gomont, 1892

Nomenclature:

Schizothrix lenormandiana

Notes: Katsiapi et al. 2012

Schizothrix simplicior Skuja, 1964

Nomenclature:

Schizothrix simplicior

Notes: Anagnostidis 1968

Schizothrix tenuis Woronichin, 1923

Nomenclature:

Schizothrix tenuis

Notes: Anagnostidis 1968

Scytonema crispum Bornet ex De Toni, 1907

Nomenclature:

Scytonema crispum st. chiastus

Notes: Anagnostidis 1968

Scytonema hofmanii var. calcicola Agardh ex Bornet \& Flahault, 1887

Nomenclature:

Scytonema hofmanii var. calcicola

Notes: Lamprinou et al. 2012

Scytonema julianum Meneghini ex B. A. Whitton, 2011

Nomenclature:

Scytonema julianum

Notes: Anagnostidis et al. 1982 
Scytonema myochrous C. Agardh ex Bornet \& Flahault, 1886

Nomenclature:

Scytonema myochrous

Notes: Anagnostidis 1968

Scytonema polycystum Bornet \& Flahault, 1886

Nomenclature:

Scytonema polycystum

Notes: Anagnostidis 1968

Scytonema wolleanum Forti, 1907

Nomenclature:

Scytonema mirabile

Notes: Anagnostidis 1968

Scytonematopsis crustacea (Thuret ex Bornet \& Flahault) Koválik \& Komárek, 1988.

Nomenclature:

Calothrix crustacea

Notes: Anagnostidis and Golubic 1966

Siphonema polonicum (Raciborski) Geitler, 1925

Nomenclature:

Siphononema polonicum

Notes: Anagnostidis 1968

Snowella atomus Komárek \& Hindák, 1988

Nomenclature:

Snowella atomus

Notes: Danielidis et al. 1996 
Snowella lacustris (Chodat) Komárek \& Hindák, 1988

Nomenclature:

Gomphosphaeria lacustris

Notes: Anagnostidis 1968

Snowella litoralis (Häyrén) Komárek \& Hindák, 1988

Nomenclature:

Snowella litoralis

Notes: Hindak and Moustaka 1988

Snowella septentrionalis Komárek \& Hindák, 1988

Nomenclature:

Snowella septentrionalis

Notes: Montesanto et al. 1999

Solentia paulocellularis (Ercegovic) LeCampion-Alsumard \& Golubic ex Belyakova, 1988

Nomenclature:

Hormathonema paulocellulare

Notes: Anagnostidis and Pantazidou 1988a

Sphaerocavum microcystiforme (Hindák) Azevedo \& Sant' Anna, 2003

Nomenclature:

Pannus microcystiformis

Notes: Hindák 1993

Sphaerospermopsis aphanizomenoides (Forti) Zapomelová, Jezberová, Hrouzek, Hisem, Reháková \& Komárková, 2010

Nomenclature:

Anabaena aphanizomenoides

Notes: Moustaka-Gouni 1988 
Spirulina caldaria Tilden, 1898

Nomenclature:

Spirulina caldaria

Notes: Anagnostidis 1961

Spirulina corakiana Playfair, 1914

Nomenclature:

Spirulina corakiana

Notes: Anagnostidis 1961

Spirulina labyrinthiformis Gomont, 1892

Nomenclature:

Spirulina labyrinthiformis

Notes: Anagnostidis and Golubic 1966

Spirulina major Kützing ex Gomont, 1892

Nomenclature:

Spirulina major

Notes: Anagnostidis 1961

Spirulina meneghiniana Zanardini ex Gomont, 1892

Nomenclature:

Spirulina meneghiniana

Notes: Anagnostidis and Golubic 1966

Spirulina princeps W. \& G. S. West, 1902

Nomenclature:

Spirulina princeps

Notes: Anagnostidis 1961

Spirulina robusta H. Welsh, 1965

Nomenclature:

Spirulina subsalsa var. crassior 
Notes: Anagnostidis 1961

Spirulina subsalsa Oersted ex Gomont, 1892

Nomenclature:

Spirulina subsalsa

Notes: Anagnostidis 1961

Spirulina subtilissima Kützing ex Gomont, 1892

Nomenclature:

Spirulina subtilissima

Notes: Anagnostidis 1961

Spirulina tenerrima Kützing ex Gomont, 1892

Nomenclature:

Spirulina tenerrima

Notes: Anagnostidis 1961

Spirulina versicolor Cohn ex Gomont, 1892

Nomenclature:

Spirulina subsalsa st. versicolor

Notes: Anagnostidis 1968

Stanieria sublitoralis (Lindstedt) Anagnostidis \& Pantazidou, 1991

Nomenclature:

Staniera sublitoralis

Notes: Anagnostidis and Pantazidou 1991a

Stanieria sphaerica (Setchel \& Gardner) Anagnostidis \& Pantazidou,1991 Nomenclature:

Dermocarpa sphaerica

Notes: Anagnostidis and Golubic 1966 
Stichosiphon pseudopolymorphus (F. E. Fritsch) Komárek, 1989

Nomenclature:

Chamaesiphon pseudopolymorphus

Notes: Anagnostidis 1968

Stigonema hormoides Bornet \& Flahault, 1886

Nomenclature:

Stigonema hormoides

Notes: Anagnostidis and Economou-Amilli 1978

Stigonema mamillosum C. Agardh ex Bornet \& Flahault, 1887

Nomenclature:

Stigonema mamillosum

Notes: Anagnostidis 1968

Stigonema minutum Hassall ex Bornet \& Flahault, 1887

Nomenclature:

Stigonema minutum

Notes: Anagnostidis 1968

Symploca dubia Gomont, 1892

Nomenclature:

Symploca dubia

Notes: Anagnostidis 1968

Symploca elegans Kützing ex Gomont, 1892

Nomenclature:

Symploca elegans

Notes: Anagnostidis 1968

Symploca hydnoides Kützing ex Gomont, 1892

Nomenclature:

Symploca hydnoides 
Notes: Anagnostidis 1968

Symploca lacrimans Claus, 1962

Nomenclature:

Symploca lacrimans

Notes: Lamprinou et al. 2012

Symploca muralis Kuitzing ex Gomont, 1892

Nomenclature:

Symploca muralis

Notes: Lamprinou et al. 2012

Symploca muscorum Gomont ex Gomont, 1892

Nomenclature:

Symploca muscorum

Notes: Anagnostidis 1968

Symploca radians (Kützing) Rabenhorst ex Gomont, 1892

Nomenclature:

Symploca radians

Notes: Lamprinou et al. 2012

Symploca thermalis Gomont, 1892

Nomenclature:

Symploca thermalis

Notes: Anagnostidis 1961

Symplocastrum penicillatum (Gomont) Anagnostidis, 2001 Nomenclature:

Schizothrix penicillata

Notes: Anagnostidis 1968 
Synechococcus salinarum Komárek, 1956

Nomenclature:

Synechoccus salinarum

Notes: Anagnostidis and Roussomoustakaki 1991

Synechococcus bigranulatus Skuja, 1933

Nomenclature:

Synechococcus elongatus $\mathrm{f}$. thermalis

Notes: Anagnostidis 1961

Synechococcus elongatus Nägeli, 1849

Nomenclature:

Synechococcus elongatus

Notes: Anagnostidis and Golubic 1966

Synechocystis aquatilis Sauvageau, 1892

Nomenclature:

Synechocystis aquatilis

Notes: Anagnostidis 1961

Synechocystis crassa Woronichin, 1929

Nomenclature:

Synechocystis crassa

Notes: Anagnostidis 1968

Synechocystis diplococca (Pringsheim) Bourrelly, 1970

Nomenclature:

Synechocystis diplococcus

Notes: Anagnostidis and Economou-Amilli 1978

Synechocystis endobiotica (Elenkin \& Hollerbach) Elenkin, 1938

Nomenclature:

Aphanocapsa endophytica 
Notes: Anagnostidis 1968

Synechocystis minuscula Woronichin, 1926

Nomenclature:

Synechocystis minuscula

Notes: Anagnostidis 1961

Synechocystis pevalekii Ercegovic 1925

Nomenclature:

Synechocystis pevalekii

Notes: Anagnostidis 1968

Synechocystis salina Wislouch, 1924

Nomenclature:

Synechocystis salina

Notes: Anagnostidis 1961

Synechocystis sallensis Skuja, 1930

Nomenclature:

Synechocystis sallensis

Notes: Metaxatos et al. 2003

Synechocystis thermalis J. J. Copeland, 1936

Nomenclature:

Synechocystis thermalis

Notes: Anagnostidis 1961

Tapinothrix crustacea (Woronichin) Bohunická \& Johansen in Bohunická, Johansen \& Fuciková, 2011

Nomenclature:

Homoeothrix crustacea

Notes: Anagnostidis 1968 
Tapinothrix varians (Geitler) Bohunická \& J. R. Johansen in Bohunická, Johansen \& Fuciková, 2011

Nomenclature:

Homoeothrix varians

Notes: Anagnostidis 1968

Tolypothrix cavernicola Weber-van Bosse, 1913

Nomenclature:

Tolypothrix cavernicola

Notes: Lamprinou et al. 2012

Tolypothrix distorta Kützing ex Bornet \& Flahault, 1886

Nomenclature:

Tolypothrix distorta

Notes: Anagnostidis 1968

Tolypothrix elenkinii Hollerbach, 1923

Nomenclature:

Tolypothrix elenkinii

Notes: Anagnostidis 1968

Tolypothrix fragilissima Ercegovic, 1925

Nomenclature:

Tolypothrix fragilissima

Notes: Lamprinou et al. 2012

Tolypothrix penicillata Thuret ex Bornet \& Flahault, 1886

Nomenclature:

Tolypothrix penicillata

Notes: Anagnostidis 1968 
Tolypothrix rivularis Hansgirg, 1892

Nomenclature:

Tolypothrix rivularis

Notes: Lamprinou et al. 2012

Toxopsis calypsus Lamprinou, Skaraki, Kotoulas, Economou-Amili \& Pantazidou, 2012

Nomenclature:

Toxopsis calypsus

Notes: Lamprinou et al. 2012

Trichocoleus delicatulus (W. \& G. S. West) Anagnostidis, 2001

Nomenclature:

Microcoleus delicatulus

Notes: Anagnostidis 1968

Trichocoleus minimus (Frémy) Anagnostidis, 2001

Nomenclature:

Microcoleus minimus

Notes: Anagnostidis et al. 1982

Trichocoleus sociatus (W. \& G. S. West) Anagnostidis, 2001

Nomenclature:

Microcoleus sociatus

Notes: Anagnostidis 1961

Trichocoleus tenerrimus (Gomont) Anagnostidis, 2001

Nomenclature:

Microcoleus tenerrimus

Notes: Anagnostidis and Economou-Amilli 1978 
Trichocoleus voukii (Frémy) Anagnostidis, 2001

Nomenclature:

Microcoleus voukii

Notes: Anagnostidis 1968

Trichodesmium cf. iwanoffianum Nygaard, 1926

Nomenclature:

Oscillatoria cf. iwanoffiana

Notes: Moustaka 1988

Trichodesmium erythraeum Ehrenberg ex Gomont, 1892

Nomenclature:

Trichodesmium erythraeum

Notes: Metaxatos et al. 2003

Trichormus azollae (Strasburger) Komárek \& Anagnostidis, 1989

Nomenclature:

Anabaena azollae

Notes: Anagnostidis et al. 1988b

Trichormus thermalis (V.Vouk) Komárek \& Anagnostidis, 1989

Nomenclature:

Anabaena thermalis

Notes: Anagnostidis 1961

Trichormus variabilis (Kützing ex Bornet \& Flahault) Komárek \& Anagnostidis, 1989

Nomenclature:

Anabaena variabilis

Notes: Anagnostidis 1968 
Woronichinia compacta (Lemmermann) Komárek \& Hindák, 1988

Nomenclature:

Woronichinia compacta

Notes: Tryfon et al. 1996

Woronichinia naegeliana (Unger) Elenkin, 1933

Nomenclature:

Coelosphaerium nägelianum

Notes: Anagnostidis 1968

Xenococcus cf. pyriformis Setchell \& N. L. Gardner in N. L. Gardner, 1918 Nomenclature:

Xenococcus cf. pyriformis

Notes: Radea et al. 2010

Xenococcus schousboei Thuret in Bornet \& Thuret, 1880

Nomenclature:

Xenococcus schousboei

Notes: Anagnostidis and Golubic 1966

Xenotholos kerneri (Hansgirg) M. Gold-Morgan, G. Montejano \& J. Komárek, 1994

Nomenclature:

Xenococcus kerneri

Notes: Anagnostidis and Golubic 1966

Yonedaella lithophila (Ercegovic) Umezaki, 1962

Nomenclature:

Sphaeronema lithophilum

Notes: Anagnostidis 1968 


\section{Analysis}

A total of 543 species and 85 infraspecific taxa have been found in Greece up to date. The species are classified in 130 genera, 41 families, and 8 orders. The orders Synechococcales and Oscillatoriales have the highest number of species (158 and 153 species, respectively), whereas these two orders along with Nostocales and Chroococcales cover $93 \%$ of the known Greek cyanobacteria species (Fig. 1). The families Oscillatoriaceae, Leptolyngbyaceae, Microcoleaceae, and Chroococcaceae have the highest number of species (>30 each) (Fig. 2a); the genera Phormidium, Leptolyngbya, Oscillatoria, Chroococcus, Aphanocapsa, and Gloeocapsa have the highest number of species (>15 each) (Fig. 2b). Fourteen species were found to include 85 infraspecific taxa ( Table 1); those infraspecific taxa were not included in the checklist. Furthermore, 21 species recorded in Greece are not valid or of uncertain taxonomic status (Table 2) and were not included in the checklist.

Table 1.

List of infraspecific cyanobacterial taxa known to occur in Greece. For each taxon the first publication mentioning its occurrence in Greece is given.

\begin{tabular}{|c|c|c|}
\hline Species & Infraspecific epithet & Reference \\
\hline Anabaena torulosa & var. tenuis & Anagnostidis 1961 \\
\hline Anabaena oscillarioides & fa. circinalis & Anagnostidis 1968 \\
\hline Aphanothece microscopica & fa. endophytica & Anagnostidis 1968 \\
\hline \multirow[t]{9}{*}{ Entophysalis deusta } & st. solentioides & Anagnostidis 1968 \\
\hline & st. dermatocarpoides & Anagnostidis 1968 \\
\hline & st. dermocarpoides & Anagnostidis 1968 \\
\hline & st. gloeocapsoides & Anagnostidis 1968 \\
\hline & st. hormathonematoides & Anagnostidis 1968 \\
\hline & st. hyelloides & Anagnostidis 1968 \\
\hline & st. pleurocapsoides & Anagnostidis 1968 \\
\hline & st. tryponematoides & Anagnostidis 1968 \\
\hline & st. typicus & Anagnostidis 1968 \\
\hline \multirow[t]{6}{*}{ Gloeocapsa biformis } & st. derm. col. ralfsianus & Anagnostidis 1968 \\
\hline & st. derm. magma & Anagnostidis 1968 \\
\hline & st. dermochrous & Anagnostidis 1968 \\
\hline & st. nannocytosus & Anagnostidis 1968 \\
\hline & st. perdurans & Anagnostidis 1968 \\
\hline & st. punctatus & Anagnostidis 1968 \\
\hline \multirow[t]{2}{*}{ Gloeocapsa compacta } & st. perdurans & Anagnostidis 1968 \\
\hline & st. lam. col. magma & Anagnostidis 1968 \\
\hline
\end{tabular}




\begin{tabular}{|c|c|c|}
\hline & st. lam. col. typicus & Anagnostidis 1968 \\
\hline & st. lam. coloratus & Anagnostidis 1968 \\
\hline & st. nannocytosus & Anagnostidis 1968 \\
\hline & st. perdurans & Anagnostidis 1968 \\
\hline & st. simplex & Anagnostidis 1968 \\
\hline \multirow[t]{4}{*}{ Gloeocapsa kützingiana } & st. lam. col. magma & Anagnostidis 1968 \\
\hline & st. lam. coloratus & Anagnostidis 1968 \\
\hline & st. perdurans & Anagnostidis 1968 \\
\hline & st. rupestris & Anagnostidis 1968 \\
\hline \multirow[t]{9}{*}{ Gloeocapsa sanguinea } & st. col. alp. magma & Anagnostidis 1968 \\
\hline & st. col. alp. ralfsianus & Anagnostidis 1968 \\
\hline & st. col. ralfsianus & Anagnostidis 1968 \\
\hline & st. lam. col. alp. magma & Anagnostidis 1968 \\
\hline & st. lam. col. alpinus & Anagnostidis 1968 \\
\hline & st. lam. col. magma & Anagnostidis 1968 \\
\hline & st. lam. coloratus & Anagnostidis 1968 \\
\hline & st. perdurans & Anagnostidis 1968 \\
\hline & st. simplex & Anagnostidis 1968 \\
\hline \multirow[t]{19}{*}{ Mastigocladus laminosus } & fa. oscillariodes subfa. subrecta & Anagnostidis 1961 \\
\hline & fa. oscillariodes subfa. circinata & Anagnostidis 1961 \\
\hline & fa. oscillariodes subfa. spiroides & Anagnostidis 1961 \\
\hline & fa. pseudanabaenoides & Anagnostidis 1961 \\
\hline & fa. pseudanabaenoides subfa. subrecta & Anagnostidis 1961 \\
\hline & fa. pseudanabaenoides subfa. circinata & Anagnostidis 1961 \\
\hline & fa. pseudanabaenoides subfa. spiroides & Anagnostidis 1961 \\
\hline & fa. lyngbyoides & Anagnostidis 1961 \\
\hline & fa. lyngbyoides subfa. subrecta & Anagnostidis 1961 \\
\hline & fa. lyngbyoides subfa. circinata & Anagnostidis 1961 \\
\hline & fa. phormidioides & Anagnostidis 1961 \\
\hline & fa. phormidioides subfa. subrecta & Anagnostidis 1961 \\
\hline & fa. phormidioides subfa. circinata & Anagnostidis 1961 \\
\hline & fa. plectonematoides & Anagnostidis 1961 \\
\hline & fa. symplocoides & Anagnostidis 1961 \\
\hline & fa. anabaenoides & Anagnostidis 1961 \\
\hline & fa. anabaenoides subfa. subrecta & Anagnostidis 1961 \\
\hline & fa. anabaenoides subfa. circinata & Anagnostidis 1961 \\
\hline & fa. anabaenoides subfa. spiroides & Anagnostidis 1961 \\
\hline
\end{tabular}




\begin{tabular}{|c|c|c|}
\hline & fa. nostocoides & Anagnostidis 1961 \\
\hline & fa. aulosiroides & Anagnostidis 1961 \\
\hline & fa. stigonematoides & Anagnostidis 1961 \\
\hline & fa. microchaetoides & Anagnostidis 1961 \\
\hline & fa. tolypotrichoides & Anagnostidis 1961 \\
\hline & fa. scytonematoides & Anagnostidis 1961 \\
\hline & fa. typica & Anagnostidis 1961 \\
\hline & fa. typica subfa. normalis & Anagnostidis 1961 \\
\hline & fa. typica subfa. brachytrichoides & Anagnostidis 1961 \\
\hline & fa. pallidus & Anagnostidis 1961 \\
\hline & fa. oscillariodes & Anagnostidis 1961 \\
\hline & fa. chroococcoides & Anagnostidis 1961 \\
\hline & fa. pleurocapsoides & Anagnostidis 1961 \\
\hline Nostoc punctiforme & var. populorum ${ }^{1}$ & Anagnostidis and Economou-Amilli 1978 \\
\hline Pseudanabaena articulata & fa. ${ }^{2}$ & Anagnostidis 1968 \\
\hline Pseudanabaena lonchoides & fa. circinata & Anagnostidis 1961 \\
\hline & fa. crassior & Anagnostidis 1968 \\
\hline & fa. tenuis & Anagnostidis 1968 \\
\hline Scytonema myochrous & st. crustaceus & Anagnostidis 1968 \\
\hline & st. petalonema & Anagnostidis 1968 \\
\hline & st. petalonema & Anagnostidis 1968 \\
\hline & st. petalonema & Anagnostidis 1968 \\
\hline & st. typicus & Anagnostidis 1968 \\
\hline Siphononema polonicum & st. chamaesiphonoides & Anagnostidis 1968 \\
\hline & st. juvenilis & Anagnostidis 1968 \\
\hline & st. scopulonematoides & Anagnostidis 1968 \\
\hline & st. stigonematoides & Anagnostidis 1968 \\
\hline Synechocystis aquatilis & var. minor & Anagnostidis 1961 \\
\hline
\end{tabular}

${ }^{1}$ The current taxonomic status is Nostoc punctiforme fa. populorum

${ }^{2}$ The author does not identify a certain forma 
Table 2.

List of cyanobacterial taxa known to occur in Greece with uncertain or not valid taxonomic status. For each taxon the publication mentioning its occurrence in Greece and the current taxonomic status is given.

\begin{tabular}{|c|c|c|}
\hline Taxon & Reference & Current taxonomic status \\
\hline Achroonema lentum & $\begin{array}{l}\text { Anagnostidis and } \\
\text { Economou-Amilli } 1980\end{array}$ & $\begin{array}{l}\text { unclassified below the Kingdom level (Guiry and Guiry } \\
\text { 2016) }\end{array}$ \\
\hline Achroonema proteiforme & Anagnostidis 1968 & \\
\hline Achroonema angustum & Anagnostidis 1968 & \\
\hline Achroonema atriculatum & Anagnostidis 1968 & \\
\hline Achroonema splendens & Anagnostidis 1968 & \\
\hline Achroonema subsalsum & Anagnostidis 1968 & \\
\hline $\begin{array}{l}\text { Desmanthos } \\
\text { thiocrenophilum (?) }\end{array}$ & Anagnostidis 1968 & \\
\hline Pelonema aphane & Anagnostidis 1968 & \\
\hline $\begin{array}{l}\text { Pelonema cf. } \\
\text { subtilissimum }\end{array}$ & $\begin{array}{l}\text { Anagnostidis and } \\
\text { Roussomoustakaki } 1991\end{array}$ & \\
\hline $\begin{array}{l}\text { Pelonema } \\
\text { pseudovacuolatum }\end{array}$ & Anagnostidis 1968 & \\
\hline Pelonema subtilissimum & Anagnostidis 1968 & \\
\hline Pelonema tenue & Anagnostidis 1968 & \\
\hline Peloploca ferruginea (?) & Anagnostidis 1968 & \\
\hline Peloploca taeniata & Anagnostidis 1968 & \\
\hline Peloploca undulata & Anagnostidis 1968 & \\
\hline Pleurocapsa crepidinum & $\begin{array}{l}\text { Anagnostidis and Golubic } \\
1966\end{array}$ & $\begin{array}{l}\text { needs revision; possibly a Chroococcidiopsis species } \\
\text { (Komárek and Anagnostidis 2005) }\end{array}$ \\
\hline $\begin{array}{l}\text { Aphanothece nidulans } \\
\text { var. nidulans }\end{array}$ & $\begin{array}{l}\text { Hindak and Moustaka } \\
1988\end{array}$ & $\begin{array}{l}\text { uncertain; Algabase (Guiry and Guiry 2016) contains only } \\
\text { two valid } A \text {. nidulans varieties: var. longissima Nash and } \\
\text { var. thermalis Hansgirg }\end{array}$ \\
\hline $\begin{array}{l}\text { Hydrocoleus } \\
\text { homoeotrichus }\end{array}$ & Anagnostidis et al. 1983 & $\begin{array}{l}\text { needs revision; sensu Anagnostidis et al 1983, probably } \\
\text { belongs to the Oscillatoriaceae according to trichome } \\
\text { structure, and into the genus Blennothrix (Komárek and } \\
\text { Anagnostidis 2005) }\end{array}$ \\
\hline $\begin{array}{l}\text { Achroonema } \\
\text { pseudangustum }\end{array}$ & $\begin{array}{l}\text { Anagnostidis and } \\
\text { Economou-Amilli } 1980\end{array}$ & not valid (Guiry and Guiry 2016) \\
\hline Scolecia filosa & Färber et al. 2015 & $\begin{array}{l}\text { Ichnotaxa produced by unknown heterotrophic organisms } \\
\text { (Heindel et al. 2009) }\end{array}$ \\
\hline $\begin{array}{l}\text { Gloeocapsa } \\
\text { polydermatica }\end{array}$ & Anagnostidis 1961 & $\begin{array}{l}\text { Sporotetras polydermatica [Chlorophyta] (Guiry and Guiry } \\
\text { 2016) }\end{array}$ \\
\hline
\end{tabular}




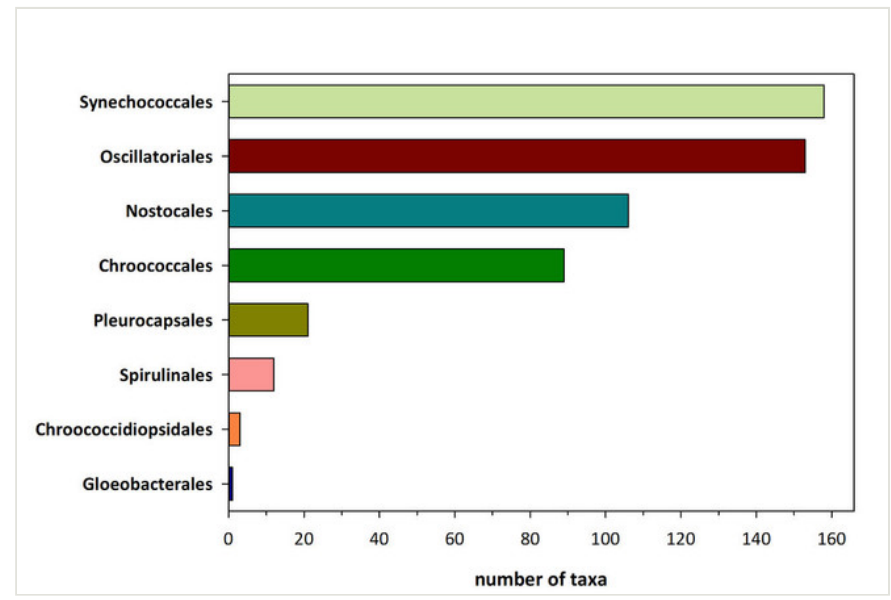

Figure 1.

Classification of cyanobacteria of Greece in orders.
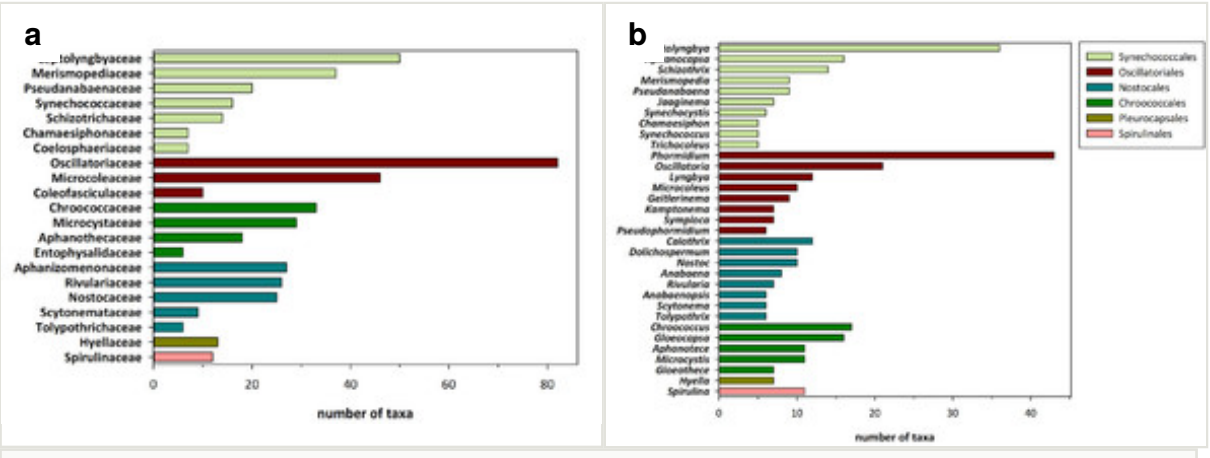

Figure 2.

Classification of cyanobacteria of Greece in families (a) and genera (b). Only families and genera including more than five species are shown.

\section{Discussion}

A thorough review of the relevant literature revealed that 543 species of cyanobacteria have been reported up to date from Greece. The species recorded from this area are classified in 130 genera and 41 families, all of the 8 orders of the class Cyanophyceae (Komárek et al. 2014) being represented. In this checklist we have not included 85 infraspecific taxa mostly from the genera Entophysalis, Gloeocapsa, Mastigocladus, Scytonema, and Siphononema recorded in several habitats such as gulfs, waterfalls, caves, and lakes by Anagnostidis (Anagnostidis 1961, Anagnostidis 1968). In the genus Mastigocladus numerous taxa were described, which do not belong to this genus (Komárek 2013). Among the formae described by Anagnostidis, some are subject to revision [e.g. M. 
fa. microchaetoides is "surely not Mastigocladus, as it is morphologically similar to Aulosira" according to Komárek (2013)]. The great morphological variability of this chroococcalean and nostocalean species should be further investigated in the future.

A number of species reported in Greece were found to be of not valid or of uncertain taxonomic status: The species Pleurocapsa crepidinum is possibly a Chroococcidiopsis species and needs revision (Komárek and Anagnostidis 2005). The species Hydrocoleus homoeotrichus sensu Anagnostidis et al. (1983), probably belongs to the Oscillatoriaceae and into the genus Blennothrix (Komárek and Anagnostidis 2005) according to its trichome structure. The taxon Aphanothece nidulans var. nidulans found in Lake Volvi (Hindak and Moustaka 1988) is not included either in the Chroococcales volume of Süsswasserflora von Mitteleuropa (Komárek and Anagnostidis 1999) nor in the AlgaeBase (Guiry and Guiry 2016); Algaebase contains only two valid $A$. nidulans varieties: var. longissima Nash and var. thermalis Hansgirg. Gloeocapsa polydermatica Kützing found in Nigrita hot springs (Anagnostidis 1961) is currently regarded as a taxonomic synonym of the chlorophyte Sporotetras polydermatica (Kützing) I.Kostikov, T.Darienko, A.Lukesová, \& L.Hoffmann (Guiry and Guiry 2016). The taxon Scolecia filosa found in microbial bioerosion at an underwater marine site in Peloponnese (Färber et al. 2015) belongs to ichnotaxa produced by unknown heterotrophic organisms (Heindel et al. 2009), not cyanobacteria. The remaining 17 species belong to the family Pelonemataceae; this family, consisting of the genera Achroonema, Pelonema, Peloploca, and Desmanthos, was created to accommodate some colorless bacteria with multicellular filaments (trichomes), which were observed in hypolimnia of lakes and ponds or in sulfur springs (Hirsch 1981). At present, members of this family are considered unclassified below the Kingdom level (Guiry and Guiry 2016).

It is worth mentioning that 18 of the species included in the list have been initially described from Greek habitats. The marine epilithic Ammatoidea aegea described from Saronikos Gulf (Aegean Sea) (Anagnostidis and Pantazidou 1991a) is considered endemic to this area (Komarek and Anagnostidis 2005). Interestingly, most of these new species were found in rarely sampled habitats, such as caves [e.g. Toxopsis calypsus, Ifinoe spelaea (Lamprinou et al. 2011)], marine coastal rocks [e.g. epilithic Cyanosarcina thalassia (Anagnostidis and Pantazidou 1991b) or endolithic Cyanosaccus aegaeus and Cyanosaccus atticus (Anagnostidis and Pantazidou 1985, Anagnostidis and Pantazidou 1988c)], archaeological sites [e.g. aerophytic, chasmoendolithic Cyanosarcina parthenonensis (Anagnostidis and Pantazidou 1991b)], heliothermal saltwork mats [Cyanothece halobia (Anagnostidis and Roussomoustakaki 1991)], and waterfalls [Phormidium edessae (Skuja 1937)], highlighting the need for further research to reveal the unknown diversity in these environments. A recent study by Bravakos et al. (2016) on cyanobacteria strains isolated from mats of thermal springs of Greece indicates at least six new taxa at the genus level. Furthermore, Konstantinou et al. (2016) report possible new species on cyanobacteria strains isolated from marine sponges from the Aegean Sea, thus corroborating the abundant diversity of lesser-known environments. 
To the best of our knowledge, the list of Greek cyanobacteria compiled for the needs of the present study is the first list of both freshwater and marine Cyanophyceae in the Mediterranean. There is another list presenting 327 species from Israel (Tsarenko et al. 2000), but this refers only to the continental part of that country. The Egyptian list (Nassar 2014) contains 19 cyanobacteria species, whereas a phytoplankton checklist from the Eastern Adriatic (Vilicic et al. 2002) contains no cyanobacteria. Other local cyanobacterial lists were published in the past 15 years from continental parts of Europe, e.g. Slovakia, Sweden, Poland, Romania, Ukraine, Slovenia, the Netherlands, and the Czech Republic (Kaštovský et al. 2010 and references therein), as well as a checklist from the Baltic Sea reporting 172 cyanobacterial species (Hällfors 2004). The Czech Republic list comprises 505 species, 392 of which are considered recently present according to Kaštovský et al. 2010. The cyanobacteria checklist of Nepal (Rai et al. 2011) presents 274 species. Therefore, it appears that Greece hosts a comparatively high diversity of cyanobacteria, suggesting that the Mediterranean area is also a hot spot for the "substantially underestimated or unexplored" (Coll et al. 2010) microbes.

\section{Acknowledgements}

This paper is dedicated to the memory of Professor Konstantinos Anagnostidis (12 December 1924-9 December 1994), whose pioneer work on cyanobacterial diversity has paved the way for modern cyanobacterial taxonomy and the study of Phycology in Greece.

This study was supported by the LifeWatchGreece Infrastructure, (MIS 384676), funded by the Greek Government under the General Secretariat of Research and Technology (GSRT), ESFRI Projects, National Strategic Reference Framework (NSRF).

SG gratefully acknowledges Prof. Eleni Voultsiadou for critically reading the manuscript, useful suggestions, and fruitful discussions. We thank Dr. Vasilis Gerovasileiou for his support.

\section{Author contributions}

SG conceived, designed and supervised this project, and prepared the manuscript. IO and NP collected the data. IO, MP, and SG and built the database.

\section{References}

- $\quad$ Anagnostidis K (1961) Investigations of the Cyanophyceae in some thermal springs in Greece. Institute of Systematic Botany and Phytogeography of the Aristotle University of Thessaloniki, Thessaloniki, 7: 1-322 pp. [In Greek].

- Anagnostidis K (1968) Investigations of Sulphur-communities (Sulphuretum) in marine and freshwater habitats. A taxonomic, floristic, ecological, phytosociological, 
phytogeographic study. 10. Scientific annals of the Faculty of Sciences, Aristotle University of Thessaloniki, Thessaloniki, 406-866 pp. [In Greek].

- Anagnostidis K (1977) Some remarks on the taxonomy of the genus Borzia Cohn ex Gom. from Greece. Schweizerische Zeitschrift für Hydrologie 39: 121-123.

- $\quad$ Anagnostidis K, Economou-Amilli A (1978) Microorganisms from the volcano of Nea Kammeni Island (Santorini). Thera and the Aegean World 1: 707-723.

- $\quad$ Anagnostidis K, Economou-Amilli A (1980) Limnological studies on Lake Pamvotis (loannina), Greece I. Hydroclimatology, phytoplankton - periphyton with special reference to the valency of some microorganisms from sulphureta as bioindicators. Archiv für Hydrobiologie 89: 313-342.

- $\quad$ Anagnostidis K, Golubic S (1966) Über die Okologie einiger Spirulina Arten. Nova Hedwigia 11: 309-335. [In German].

- $\quad$ Anagnostidis K, Komárek J (1985) Modern approach to the classification system of cyanophytes. 1- Introduction. Arch. Hydrobiol. Suppl. 71: 291-302.

- $\quad$ Anagnostidis K, Komárek J (1988) Modern approach to the classification system of cyanophytes. 3- Oscillatoriales. Archiv für Hydrobiologie, Supplement 80: 327-472.

- Anagnostidis K, Komárek J (1990) Modern approach to the classification system of cyanophytes. 5- Stigonematales. Archiv für Hydrobiologie, Supplement 86: 1-73.

- $\quad$ Anagnostidis K, Pantazidou A (1985) Cyanosaccus aegaeus n. sp., a new marine endolithic cyanophyte from the Aegean Sea, Hellas (Greece). Archiv für Hydrobiologie (Algological Studies) 38/39 (suppl. 71): 105-114.

- $\quad$ Anagnostidis K, Pantazidou A (1988a) Endolithic cyanophytes from the saline thermal springs of Aedipsos, Hellas (Greece). Algological Studies/Archiv für Hydrobiologie, Supplement 50-53 (suppl. 80): 555-559.

- $\quad$ Anagnostidis K, Pantazidou A (1988b) Hyella kalligrammos sp. nov., Hyella maxima (Geitl.) comb. nov., and other freshwater morphotypes of the genus Hyella Born. et Flah. (Chroococcales, Cyanophyceae). Algological Studies/Archiv für Hydrobiologie, Supplement 50-53 (suppl. 80): 227-247.

- Anagnostidis K, Pantazidou A (1988c) Cyanosaccus atticus, a new marine euendolithic chroococcoid cyanophyte in relation to the epilithic Podocapsa Erceg. Algological Studies/Archiv für Hydrobiologie, Supplement 48 (suppl. 78): 279-302.

- Anagnostidis K, Pantazidou A (1991a) Ammatoidea aegaea (Oscillatoriales), a new marine epilithic species from the Aegean Sea, Hellas, with a reference to the validity of the genus Ammatoidea . Algological Studies/Archiv für Hydrobiologie, Supplement 64: 281-297.

- $\quad$ Anagnostidis K, Pantazidou A (1991b) Marine and aerophytic Cyanosarcina, Stanieria and Pseudocapsa (Chroococcales) species from Hellas (Greece). Algological Studies/ Archiv für Hydrobiologie, Supplement 64: 141-157.

- $\quad$ Anagnostidis K, Roussomoustakaki M (1988) Cyanophytes from metal burdened substrates. Algological Studies/Archiv für Hydrobiologie, Supplement 50-53 (suppl. 80): 561-564.

- $\quad$ Anagnostidis K, Roussomoustakaki M (1991) Isocystis halobia spec. nova, a benthic nostocalean cyanophyte from the heliothermal saltwork mats of Messolongion, Hellas (Greece). Algological Studies 64: 299-332.

- $\quad$ Anagnostidis K, Economou-Amilli A, Makris K (1988a) On the morphotypes of Phormidium boryanum (Bory ex Gom.) Anagn. et Kom. and Phormidium janthiphorum 
(Fiori-Mazz. ex Gom.) Elenk. A taxonomic consideration. Algological Studies/Archiv für Hydrobiologie, Supplement 50-53 (suppl. 80): 71-91.

- Anagnostidis K, Economou-Amilli A, Overbeck J (1988b) Anabaena azollae Strasb. and the periphyton of Azolla filiculoides Lam. in lake Trichonis and the lagoon of Aetolikon, Hellas (Greece). Archiv für Hydrobiologie. 133 (3): 321-343.

- Anagnostidis K, Economou-Amilli A, Pantazidou A (1982) Studies on microflora of Perama Cave, loannina, Greece. Bulletin de la Société Spéléologique de Grèce 18 (1-2): 458-530.

- $\quad$ Anagnostidis K, Economou-Amilli A, Roussomoustakaki M (1983) Epilithic and chasmolithic microflora (Cyanophyta, Bacillariophyta) from marbles of the ParthenonAcropolis, Athens, Greece. Nova Hedwigia 38: 227-288.

- Anagnostidis K, Economou-Amilli A, Tsangridis A (1981) Taxonomic and floristic studies of algae from rice-fields of Kalochorion-Thessaloniki, Greece. Nova Hedwigia 34: 1-66.

- $\quad$ Ananiadis Cl (1956) Limnological study of Lake Karla. Bulletin de L'Institut Océanographique 1083: 1-19.

- $\quad$ Bailly N, Gerovasileiou V, Arvanitidis C, Legakis A (2016) Introduction to the Greek Taxon Information System (GTIS) in LifeWatchGreece: the construction of the Preliminary Checklists of Species of Greece. Biodiversity Data Journal in press: e.

- Batler S (1998) Homer's lliad. Orange Street Press Classics, 495 pp. URL: http:// sparks.eserver.org/books/iliad.pdf

- $\quad$ Bravakos P, Kotoulas G, Skaraki K, Pantazidou A, Economou-Amilli A (2016) A polyphasic taxonomic approach in isolated strains of Cyanobacteria from thermal springs of Greece. Molecular Phylogenetics and Evolution 98: 147-160. https:// doi.org/10.1016/i.ympev.2016.02.009

- $\quad$ Christodoulou M, Meletiou-Christou M, Parmakelis A, Economou-Amilli A, Pantazidou A (2015) Further findings from Daveli Cave (Attica, Greece) enhancing the establishment of the genusOculatella (Pseudanabaenaceae, Cyanobacteria). Phytotaxa 202 (3): 169. https://doi.org/10.11646/phytotaxa.202.3.1

- Coll M, Piroddi C, Steenbeek J, Kaschner K, Rais Lasram FB, Aguzzi J, Ballesteros E, Bianchi CN, Corbera J, Dailianis T, Danovaro R, Estrada M, Froglia C, Galil B, Gasol J, Gertwagen R, Gil J, Guilhaumon F, Kesner-Reyes K, Kitsos M, Koukouras A, Lampadariou N, Laxamana E, la Cuadra CLd, Lotze H, Martin D, Mouillot D, Oro D, Raicevich S, Rius-Barile J, Saiz-Salinas JI, Vicente CS, Somot S, Templado J, Turon X, Vafidis D, Villanueva R, Voultsiadou E (2010) The Biodiversity of the Mediterranean Sea: Estimates, Patterns, and Threats. PLoS ONE 5 (8): e11842. https://

doi.org/10.1371/journal.pone.0011842

- Danielidis DB, Spartinou M, Economou-Amilli A (1996) Limnological survey of Lake Amvrakia, western Greece. Hydrobiologia 318 (3): 207-218. https://doi.org/10.1007/ $\underline{\text { bf00016682 }}$

- Duval B, Duval E, Hoham RW (1999) Snow algae of the Sierra Nevada, Spain, and High Atlas mountains of Morocco. International microbiology : the official journal of the Spanish Society for Microbiology 2 (1): 39-42.

- Economou-Amilli A, Anagnostidis K, Roussomoustakaki M (1984) Structural Aspects of the Adaptation of Some Blue-Green Algae and Diatoms to Desiccation. Tasks for vegetation science. https://doi.org/10.1007/978-94-009-6578-2 13

- Färber C, Wisshak M, Pyko I, Bellou N, Freiwald A (2015) Effects of Water Depth, Seasonal Exposure, and Substrate Orientation on Microbial Bioerosion in the Ionian 
Sea (Eastern Mediterranean). PLOS ONE 10 (4): e0126495. https://doi.org/10.1371/ journal.pone.0126495

- $\quad$ Gkelis S, Panou M (2016) Capturing biodiversity: linking a cyanobacteria culture collection to the "scratchpads" virtual research environment enhances biodiversity knowledge. Biodiversity data journal 4: e7965. https://doi.org/10.3897/BDJ.4.e7965

- $\quad$ Gkelis S, Zaoutsos N (2014) Cyanotoxin occurrence and potentially toxin producing cyanobacteria in freshwaters of Greece: A multi-disciplinary approach. Toxicon 78: 1-9. https://doi.org/10.1016/j.toxicon.2013.11.010

- $\quad$ Gkelis S, Lanaras T, Sivonen K (2015a) Cyanobacterial Toxic and Bioactive Peptides in Freshwater Bodies of Greece: Concentrations, Occurrence Patterns, and Implications for Human Health. Marine Drugs 13 (10): 6319-6335. https://doi.org/10.3390/ md13106319

- $\quad$ Gkelis S, Tussy PF, Zaoutsos N (2015b) Isolation and preliminary characterization of cyanobacteria strains from freshwaters of Greece. Open Life Sciences 10 (1): 52-60. htt ps://doi.org/10.1515/biol-2015-0006

- $\quad$ Gkelis S, Moustaka-Gouni M, Sivonen K, Lanaras T (2005) First report of the cyanobacterium Aphanizomenon ovalisporum Forti in two Greek lakes and cyanotoxin occurrence. Journal of Plankton Research 27 (12): 1295-1300. https://doi.org/10.1093/ plankt/fbi085

- $\quad$ Gkelis S, Papadimitriou T, Zaoutsos N, Leonardos I (2014) Anthropogenic and climateinduced change favors toxic cyanobacteria blooms: Evidence from monitoring a highly eutrophic, urban Mediterranean lake. Harmful Algae 39: 322-333. https:// doi.org/10.1016/..hal.2014.09.002

- $\quad$ Gkelis S, Rajaniemi P, Vardaka E, Moustaka-Gouni M, Lanaras T, Sivonen K (2005) Limnothrix redekei (Van Goor) Meffert (Cyanobacteria) Strains from Lake Kastoria, Greece Form a Separate Phylogenetic Group. Microbial Ecology 49 (1): 176-182. http s://doi.org/10.1007/s00248-003-2030-7

- $\quad$ Guiry MD, Guiry MD (2016) AlgaeBase. World-wide electronic publication, National University of Ireland, Galway. http://www.algaebase.org. Accession date: 2016716.

- Hällfors G (2004) Checklist of Baltic Sea Phytoplankton Species. 95. Baltic Sea Environment Proceedings, $210 \mathrm{pp}$.

- $\quad$ Heindel K, Westphal H, Wisshak M (2009) Data report: bioerosion in the reef framework, IODP Expedition 310 off Tahiti (Tiarei, Maraa, and Faaa sites). Proceedings of the IODP, epub pp. https://doi.org/10.2204/iodp.proc.310.201.2009

- Hindak F, Moustaka M (1988) Planktic cyanophytes of Lake Volvi, Greece. Algological Studies/Archiv für Hydrobiologie, Supplement Volumes 50-53: 497-528.

- Hindák F (1988) Taxonomic position of two cyanophyte genera, Cyanostylon Geitler and Siphonosphaera gen. nov. Archiv für Hydrobiologie/Algological Studies 78: 411-423.

- Hindák F (1993) To the taxonomy of the chroococcal genus Pannus Hickel 1991 (Cyanophyta/Cyanobacteria). Archiv für Hydrobiologie/Algological Studies 69: 1-10.

- Hirsch P (1981) The Family Pelonemataceae. The Prokaryotes. https:// doi.org/10.1007/978-3-662-13187-9 26

- Kaštovský J, Hauer T, Komarek J, Skacelova O (2010) The list of cyanobacterial species of the Czech Republic to the end of 2009. Fottea 10: 245-249. https:// doi.org/10.5507/fot.2010.015 
- $\quad$ Katsiapi M, Mazaris A, Charalampous E, Moustaka-Gouni M (2012) Watershed land use types as drivers of freshwater phytoplankton structure. Hydrobiologia 698 (1): 121-131. https://doi.org/10.1007/s10750-012-1095-z

- Katsiapi M, Moustaka-Gouni M, Michaloudi E, Kormas K (2011) Phytoplankton and water quality in a Mediterranean drinking-water reservoir (Marathonas Reservoir, Greece). Environmental Monitoring and Assessment 181: 563-575. https:// doi.org/10.1007/s10661-010-1851-3

- Komárek J (2013) Phenotypic characters of heterocytous cyanobacteria. Süßwasserflora von Mitteleuropa, Bd. 19/3: Cyanoprokaryota. https:// doi.org/10.1007/978-3-8274-2737-3 2

- Komárek J (2016) Review of the cyanobacterial genera implying planktic species after recent taxonomic revisions according to polyphasic methods: state as of 2014. Hydrobiologia 764 (1): 259-270. https://doi.org/10.1007/s10750-015-2242-0

- Komárek J, Anagnostidis K (1986) Modern approach to the classification system of cyanophytes. 2- Chroococcales. Archiv für Hydrobiologie, Supplement Volumes 73: 157-226.

- Komárek J, Anagnostidis K (1989) Modern approach to the classification system of cyanophytes. 4- Nostocales. Archiv für Hydrobiologie, Supplement 82: 247-345.

- Komárek J, Anagnostidis K (1999) Cyanoprokaryota 1. Teil: Chroococcales. Süsswasserflora von Mitteleuropa., 19/1. Spektrum Akademischer Verlag, 548 pp.

- Komárek J, Anagnostidis K (2005) Cyanoprokaryota 2. Teil/ 2nd Part: Oscillatoriales. In: Büdel B, Krienitz L, Gärtner G, Schagerl M (Eds) Süsswasserflora von Mitteleuropa, 19/2. Elsevier/Spektrum, Heidelberg, Germany, 759 pp. [ISBN 978-3827409195]

- Komárek J, Kaštovský J, Mareš J, Johansen JR (2014) Taxonomic classification of cyanoprokaryotes (cyanobacterial genera) 2014, using a polyphasic approach. Preslia 86: 295-335.

- $\quad$ Konstantinou D, Gerovasileiou V, Voultsiadou E, Gkelis S (2016) 41st CIESM Congress Proceedings. 41st CIESM Congress. 1 pp.

- Koureas D, Hardisty A, Vos R, Agosti D, Arvanitidis C, Bogatencov P, Buttigieg PL, Jong Yd, Horvath F, Gkoutos G, Groom Q, Kliment T, Kõljalg U, Manakos I, Marcer A, Marhold K, Morse D, Mergen P, Penev L, Pettersson L, Svenning J, de Putte Av, Smith V (2016) Unifying European Biodiversity Informatics (BioUnify). Research Ideas and Outcomes 2: e7787. https://doi.org/10.3897/rio.2.e7787

- Lamprinou V, Economou-Amilli A, Danielidis D, Pantazidou A (2012) Distribution survey of Cyanobacteria in three Greek caves of Peloponnese. International Journal of Speleology 41 (2): 267-272. https://doi.org/10.5038/1827-806x.41.2.12

- Lamprinou V, Danielidis D, Pantazidou A, Oikonomou A, Economou-Amilli A (2014) The show cave of Diros vs. wild caves of Peloponnese, Greece - distribution patterns of Cyanobacteria. International Journal of Speleology 43 (3): 335-342. https:// doi.org/10.5038/1827-806x.43.3.10

- Lamprinou V, Pantazidou A, Papadogiannaki G, Radea C, Economou-Amilli A (2009) Cyanobacteria and associated invertebrates in Leontari Cave. Fottea 9: 155-164. https:// doi.org/10.5507/fot.2009.014

- Lamprinou V, Hernandez-Marine M, Canals T, Kormas K, Economou-Amilli A, Pantazidou A (2011) Morphology and molecular evaluation of Iphinoe spelaeobios gen. nov., sp. nov. and Loriellopsis cavernicola gen. nov., sp. nov., two stigonematalean cyanobacteria from Greek and Spanish caves. INTERNATIONAL JOURNAL OF 
SYSTEMATIC AND EVOLUTIONARY MICROBIOLOGY 61 (12): 2907-2915. https:// doi.org/10.1099/ijs.0.029223-0

- $\quad$ Lamprinou V, Hernandez-Marine M, Pachiadaki M, Kormas K, Economou-Amilli A, Pantazidou A (2013a) New findings on the true-branched monotypic genus Iphinoe (Cyanobacteria) from geographically isolated caves (Greece). Fottea 13: 15-23. https:// doi.org/10.5507/fot.2013.002

- Lamprinou V, Skaraki K, Kotoulas G, Anagnostidis K, Economou-Amilli A, Pantazidou A (2013b) A new species of Phormidium (Cyanobacteria, Oscillatoriales) from three Greek Caves: morphological and molecular analysis. Fundamental and Applied Limnology / Archiv für Hydrobiologie 182 (2): 109-116. https://doi.org/10.1127/1863-9135/2013/0323

- Lanaras T, Tsitsamis S, Chlichlia C, Cook CM (1989) Toxic cyanobacteria in Greek freshwaters. Journal of Applied Phycology 1 (1): 67-73. https://doi.org/10.1007/ $\underline{\text { bf00003537 }}$

- Metaxatos A, Panagiotopoulos C, Ignatiades L (2003) Monosaccharide and aminoacid composition of mucilage material produced from a mixture of four phytoplanktonic taxa. Journal of Experimental Marine Biology and Ecology 294 (2): 203-217. https:// doi.org/10.1016/s0022-0981(03)00269-7

- Montesanto B, Ziller S, Danielidis D, Economou-Amilli A (1999) Phytoplankton community structure in the lower reaches of a Mediterranean river (Aliakmon, Greece). Fundamental and Applied Limnology 147 (2): 171-191. https://doi.org/10.1127/archivhydrobiol/147/1999/171

- Moustaka-Gouni M (1988) The structure and dynamics of the phytoplankton assemblages in Lake Volvi, Greece I. Phytoplankton composition and abundance during the period March 1984-March 1985. Archiv für Hydrobiologie 112: 251-264.

- Moustaka-Gouni M (1993) Phytoplankton succession and diversity in a warm monomictic, relatively shallow lake: Lake Volvi, Macedonia, Greece. Intermediate Disturbance Hypothesis in Phytoplankton Ecology. https://

doi.org/10.1007/978-94-017-1919-3 5

- Moustaka-Gouni M, Nikolaidis G (1990) Phytoplankton of a warm monomictic lake Lake Vegoritis, Greece. Archiv für Hydrobiologie 119: 299-313.

- Moustaka-Gouni M, Nikolaidis G (1992) Phytoplankton and physical-chemical features of Tavropos Reservoir, Greece. Hydrobiologia 228 (2): 141-149. https://doi.org/10.1007/ bf00006202

- Moustaka-Gouni M, Albanakis K, Mitrakas M, Psilovikos A (2000) Planktic autotrophs and environmental conditions in the newly-formed hydroelectric Thesaurus reservoir, Greece. Fundamental and Applied Limnology 149 (3): 507-526. https://doi.org/10.1127/ archiv-hydrobiol/149/2000/507

- Moustaka-Gouni M, Michaloudi E, Katsiapi M, Genitsaris S (2007) Greece : The coincidence of an Arthrospira -Anabaenopsis bloom and the mass mortality of birds in Lake Koronia. Harmful algae news 35: 6-7.

- Moustaka-Gouni M, Kormas K, Vardaka E, Katsiapi M, Gkelis S (2009) Raphidiopsis mediterranea Skuja represents non-heterocytous life-cycle stages of Cylindrospermopsis raciborskii (Woloszynska) Seenayya et Subba Raju in Lake Kastoria (Greece), its type locality: Evidence by morphological and phylogenetic analysis. Harmful Algae 8 (6): 864-872. https://doi.org/10.1016/.j.hal.2009.04.003

- Moustaka-Gouni M, Kormas KA, Polykarpou P, Gkelis S, Bobori DC, Vardaka E (2010) Polyphasic evaluation of Aphanizomenon issatschenkoi and Raphidiopsis mediterranea 
in a Mediterranean lake. Journal of Plankton Research 32 (6): 927-936. https:// doi.org/10.1093/plankt/fbq019

- Moustaka-Gouni M, Hiskia A, Genitsaris S, Katsiapi M, Manolidi K, Zervou S, Christophoridis C, Triantis T, Kaloudis T, Orfanidis S (2016) First report of Aphanizomenon favaloroi occurrence in Europe associated with saxitoxins and a massive fish kill in Lake Vistonis, Greece. Marine and Freshwater Research 67: 1-8. htt ps://doi.org/10.1071/mf16029

- Moustaka M (1988) SEASONAL VARIATIONS, ANNUAL PERIODICITY AND SPATIAL DISTRIBUTION OF PHYTOPLANKTON IN LAKE VOLVI. Aristotle University of Thessaloniki, 363 pp. [In Greek].

- Nassar M (2014) Checklist of Phytoplankton Species in the Egyptian Waters of the Red Sea and Some Surrounding Habitats (1990-2010). Annual Research \& Review in Biology 4 (23): 3566-3585. https://doi.org/10.9734/arrb/2014/10866

- Overbeck J, Anagnostidis K, Economou-Amilli A (1982) A limnological survey of three Greek lakes, Trichonis, Lyssimachia and Amvrakia. Archiv fur Hydrobiologie 95: 365-394.

- $\quad$ Pantazidou A, Louvrou I, Economou-Amilli A (2006) Euendolithic shell-boring cyanobacteria and chlorophytes from the saline lagoon Ahivadolimni on Milos Island, Greece. European Journal of Phycology 41 (2): 189-200. https:// doi.org/10.1080/09670260600649420

- $\quad$ Papadimitriou T, Katsiapi M, Kormas KA, Moustaka-Gouni M, Kagalou I (2013) Artificially-born "killer" lake: Phytoplankton based water quality and microcystin affected fish in a reconstructed lake. Science of The Total Environment 452-453: 116-124. http s://doi.org/10.1016/j.scitotenv.2013.02.035

- Peck AL (1970) Aristotle. History of Animals. Books IV-VI. English translation, Introduction and Comments. Cambridge, Massachusetts: Harvard University Press., $409 \mathrm{pp}$.

- $\quad$ Radea C, Louvrou I, Pantazidou A, Amilli AE (2010) Photosynthetic microorganisms as epibionts and euendoliths on biotic substrates in a thermal spring with ferric-iron deposits. Fottea 10: 129-140. https://doi.org/10.5507/fot.2010.007

- $\quad$ Rai SK, Rai RK, Jha S (2011) Cyanobacteria of Nepal: A Checklist with Distribution. Our Nature 8 (1): 336-354. https://doi.org/10.3126/on.v8i1.4342

- $\quad$ Skuja H (1937) Süsswasseralgen aus Griechenland und Kleinasien. Hedwigia 77: 15-73. [In German].

- Stanković S (1931) Sur les particularités limnologiques de lacs égéens. Verhandlungen des Internationalen Verein Limnologie 5: 158-196.

- Tafas T, Economou-Amilli A (1997) Limnological survey of the warm monomictic lake Trichonis (central western Greece)II. Seasonal phytoplankton periodicity - a community approach . Hydrobiologia 344: 141-153. https://doi.org/10.1023/a:1002962513146

- Temponeras M, Kristiansen J, Moustaka-Gouni M (2000) Seasonal variation in phytoplankton composition and physical-chemical features of the shallow Lake Doïrani, Macedonia, Greece. The Trophic Spectrum Revisited. https://

doi.org/10.1007/978-94-017-3488-2 10

- Tryfon E (1996) Pannus spumosus (Chroococcales, Cyanoprocaryota) from Lake Mikri Prespa, Greece. Phycologia 35 (3): 222-224. https://doi.org/10.2216/ 
- Tryfon E, Moustaka-Gouni M, Nikolaidis G (1996) Phytoplankton and Nutrients in the River Strymon, Greece. Internationale Revue der gesamten Hydrobiologie und Hydrographie 81 (2): 281-292. https://doi.org/10.1002/iroh.19960810210

- Tryfon E, Moustaka-Gouni M, Nikolaidis G (1997) Planktic cyanophytes and their ecology in the shallow Lake Mikri Prespa, Greece. Nordic Journal of Botany 17 (4): 439-448. https://doi.org/10.1111/j.1756-1051.1997.tb00340.x

- Tsarenko PM, Vinogradova ON, Stupina VV, Wasser S, Nevo E (2000) Diversity of algae in the continental part of Israel. International Journal on Algae 2 (4): 20-39. http s://doi.org/10.1615/interjalgae.v2.i4.20

- Vardaka E, Moustaka-Gouni M, Lanaras T (2000) Temporal and spatial distribution of planktic cyanobacteria in Lake Kastoria, Greece, a shallow, urban lake. Nordic Journal of Botany 20 (4): 501-511. https://doi.org/10.1111/j.1756-1051.2000.tb01594.x

- Vardaka E, Moustaka-Gouni M, Cook C, Lanaras T (2005) Cyanobacterial blooms and water quality in Greek waterbodies. Journal of Applied Phycology 17 (5): 391-401. http s://doi.org/10.1007/s10811-005-8700-8

- Vareli K, Briasoulis E, Pilidis G, Sainis I (2009) Molecular confirmation of Planktothrix rubescens as the cause of intense, microcystin-Synthesizing cyanobacterial bloom in Lake Ziros, Greece. Harmful Algae 8 (3): 447-453. https://doi.org/10.1016/ j.hal.2008.09.005

- Vilicic D, Marasovic I, Miokovic D (2002) Checklist of phytoplankton in the eastern Adriatic Sea. Acta Bot. Croat. 61: 57-91.

- Voultsiadou E (2007) Sponges: an historical survey of their knowledge in Greek antiquity. Journal of the Marine Biological Association of the UK 87 (6): 1757-1763. http s://doi.org/10.1017/s0025315407057773 